\title{
A Reconciliation of Packed Column Permeability Data: Column Permeability as a Function of Particle Porosity
}

\author{
Hubert M. Quinn \\ The Wrangler Group LLC, 40 Nottinghill Road, Brighton, MA 02135, USA \\ Correspondence should be addressed to Hubert M. Quinn; hubert@wranglergroup.com
}

Received 14 November 2013; Accepted 2 January 2014; Published 1 April 2014

Academic Editor: Carmen Alvarez-Lorenzo

Copyright ( 2014 Hubert M. Quinn. This is an open access article distributed under the Creative Commons Attribution License, which permits unrestricted use, distribution, and reproduction in any medium, provided the original work is properly cited.

\begin{abstract}
In his textbook teaching of packed bed permeability, Georges Guiochon uses mobile phase velocity as the fluid velocity term in his elaboration of the Darcy permeability equation. Although this velocity frame makes a lot of sense from a thermodynamic point of view, it is valid only with respect to permeability at a single theoretical boundary condition. In his more recent writings, however, Guiochon has departed from his long-standing mode of discussing permeability in terms of the Darcy equation and has embraced the well-known Kozeny-Blake equation. In this paper, his teaching pertaining to the constant in the Kozeny-Blake equation is examined and, as a result, a new correlation coefficient is identified and defined herein based on the velocity frame used in his teaching. This coefficient correlates pressure drop and fluid velocity as a function of particle porosity. We show that in their experimental protocols, Guiochon et al. have not adhered to a strict material balance of permeability which creates a mismatch of particle porosity and leads to erroneous conclusions regarding the value of the permeability coefficient in the KozenyBlake equation. By correcting the experimental data to properly reflect particle porosity we reconcile the experimental results of Guiochon and Giddings, resulting in a permeability reference chart which is presented here for the first time. This reference chart demonstrates that Guiochon's experimental data, when properly normalized for particle porosity and other related discrepancies, corroborates the value of 267 for the constant in the Kozeny-Blake equation which was derived by Giddings in 1965.
\end{abstract}

\section{Introduction}

The value of the constant of proportionality between pressure gradient and fluid flow velocity in a column packed with granular material and how it relates to column porosity have been controversial topics for some time [1]. This is especially true in the field of chromatography because columns packed with porous particles have, at least for the last 50 years, been the main vehicle by which chromatographic separations have been carried out, and, thus, this feature has added a new dimension to the controversy. The complication arises, in part, because of the difficulty of differentiating between the free space between the particles and the free space within the particles in columns packed with porous particles; a problem that does not exist in the case of columns filled with nonporous particles.

More recently, because current chromatographic analytical and purification applications are being driven by the need for faster and faster separations, the relationship between pressure gradient and high rates of fluid velocity has taken on a special significance [2]. For example, it is now common to see the use of very small particles (circa 2 micrometers) in combination with very large operating column pressures (circa 15,000 psi) [3]. Because of the obvious impact of high operating pressure on the friability of chromatographic support particles, this development has created a new focus on the design criteria for packed columns, especially with regard to the fractional porosities within the columns. It will be appreciated that as the internal porosity of chromatographic support particles increases, the particles' ability to withstand high pressures decreases. This physical reality would seem to dictate that chromatographic support particles suitable for this newly defined operational regime of high column pressures ought to have a lower particle porosity than those in common use for conventional column pressures. Paradoxically, however, one finds examples in the 
recent literature pertaining to fully porous particles where low values for the constant of proportionality in the KozenyBlake equation are being justified on the basis of particle porosities which purportedly run counter to this principle [4]. Clearly, something is wrong.

The value of the permeability coefficient dictated by the necessity to balance the pressure gradient/fluid flow equation after all independent variables are accounted for (and which presumably has its origin in the fundamental mechanisms which underlie the generation of pressure in the first place) is by no means self-evident. From the standpoint of first principles, it would appear that its value ought to be constant regardless of the physical parameters comprising the fluid flow apparatus. However, no fully developed theoretical explanation for any constant of permeability has yet been published. On the other hand, the literature is replete with discussions of the value for this parameter which purportedly are justified on the basis of empirical data. The problem is that the authors of this literature do not agree with each other and sometimes the same author disagrees with himself. In the last few years, Georges Guiochon, in particular, has published conflicting values for this parameter, all of which are supposedly based upon measured values, yet he has made no serious effort to reconcile the conflicts or to provide any plausible underlying rationale for their differences.

As we discuss below, there are several inherent flaws in Guiochon's teaching of permeability which are at the root of his conflicting values for the coefficient. We suggest that these flaws originate in the fact that his teaching does not distinguish between the respective impacts on column permeability of (a) mass transfer by convection versus mass transfer by diffusion and (b) spherical particles versus particles having an irregular morphology. Accordingly, without substantial refinement and adjustment, Guiochon's teaching of column permeability, at best, does little to overcome the shortcomings of Darcy's law and, at worst, adds fuel to the fire of the aforementioned controversy.

\section{Guiochon's Textbook Teaching}

In his influential textbook, Fundamentals of Preparative and Nonlinear Chromatography [5], Guiochon (together with his coauthors) bases his teaching of the pressure/flow relationship in streamline flow on the Darcy equation which he displays therein as his equation (5.26b); a rearrangement of which we repeat here:

$$
\frac{\Delta P}{L}=\frac{\mu_{t} \eta}{k_{0} d_{p m}^{2}}
$$

where $\Delta P=$ pressure drop along column, $L=$ length of column, $\mu_{t}=$ mobile phase velocity, $\eta=$ absolute viscosity of fluid, $d_{p m}=$ average particle diameter, and $k_{0}=$ Guiochon's residual permeability coefficient.

All terms are self-consistent from a unit-of-measure perspective.

Note that Guiochon does not discriminate in his expression of the Darcy equation between spherical and irregular particles. In other words, in his statement of the equation, a spherical particle with a measured diameter of $x \mathrm{~cm}$ is equivalent to an irregularly shaped particle of the same measured diameter. Peculiarly, however, he goes on to say that "traditionally, a value of $k_{0}=1 \times 10^{-3}$ is used for irregular particles and $1.2 \times 10^{-3}$ for spherical ones" [5, p. 153].

Similarly, without including any porosity dependence term in the pressure/fluid flow relationship or specifying any particular relationship between column pressure gradient and column porosity for columns packed with either porous or nonporous particles, he states that $k_{0}$ varies "between $5 \times$ $10^{-4}$ and $2 \times 10^{-3}$, depending upon the compactness of the packed bed, that is, on the external porosity" [5, p. 153].

In essence, then, Guiochon bundles the variables of particle morphology and column porosity into his residual permeability coefficient, $k_{0}$. Indeed, this is why we call it his residual permeability coefficient.

In order to explore these implicit terms which are embedded in Guiochon's teaching for his $k_{0}$, we turn, for comparison purposes, to the well-known Kozeny-Blake equation:

$$
\frac{\Delta P}{L}=\frac{P_{0}\left(1-\varepsilon_{0}\right)^{2} \mu_{s} \eta}{\varepsilon_{0}^{3} d_{p}^{2}}
$$

where $P_{0}=$ the Kozeny/Blake permeability coefficient, $\varepsilon_{0}=$ external porosity of column, $\mu_{s}=$ fluid superficial velocity, and $d_{p}=$ spherical particle diameter equivalent.

As we noted above, Guiochon acknowledges in his teaching that particle irregularity does influence pressure drop. In a modification of the Kozeny-Blake equation proposed by Carman, this factor was expressly accounted for by adding a parameter to represent particle sphericity, which we denote with the symbol $\Omega_{p}$ (Carman gave it the symbol $\phi$ in his original publication [6]). It will be appreciated that the value of $\Omega_{p}$ is equal to 1 for spherical particles and is less than 1 for particles having an irregular morphology. Accordingly, the Kozeny-Blake equation (as modified by Carman) ${ }^{1}$ is expressed as

$$
\frac{\Delta P}{L}=\frac{P_{0}\left(1-\varepsilon_{0}\right)^{2} \mu_{s} \eta}{\varepsilon_{0}^{3}\left(d_{p m} \Omega_{p}\right)^{2}},
$$

where $\Omega_{p}=$ Carman's particle adjustment factor for deviations from perfect sphericity and $d_{p m} \Omega_{p}=d_{p}$.

As for column porosity, note that the Kozeny-Blake equation includes a combination of porosity terms which, taken together, can be viewed as its porosity dependence parameter, which we notate with the symbol $\Psi_{o}$ :

$$
\Psi_{0}=\frac{\left(1-\varepsilon_{0}\right)^{2}}{\varepsilon_{0}^{3}},
$$

where $\varepsilon_{0}=$ the volume of free space between the particles in the column/the volume of the empty column.

Comparing (1) and (3), the reader will observe that there still is another difference; the fluid velocity frames are different. Accordingly, in order to establish the relationship between the values of Kozeny-Blake's $P_{0}$ and the value of Guiochon's $k_{0}$, one needs to delineate the impact of 
Guiochon's velocity frame upon his permeability coefficient. Before attempting to do this, a review of the various velocity frames typically used to characterize flow in porous media may be helpful.

\section{Fluid Velocity Frames}

3.1. Superficial Velocity. The fluid velocity term in both the Darcy equation and the Kozeny-Blake equation is the volumetric flow rate of the fluid divided by the cross-sectional area of the empty column. This velocity is known as the "average fluid superficial linear velocity" or, simply, the "superficial velocity" and is defined as follows:

$$
\mu_{s}=\frac{\mathrm{Q}}{\mathrm{A}}
$$

where $Q=$ volumetric flow rate, $A=$ cross-sectional area of the column, and $\mu_{s}=$ superficial velocity.

Thus, superficial velocity is not influenced in any way by the existence or structure of the packed bed. Furthermore, it does not change depending upon whether the particles in the bed are porous or nonporous.

3.2. Interstitial Velocity. The convective fluid velocity between the particles of a packed bed is called the "average fluid interstitial linear velocity" or, simply, the "interstitial velocity." Interstitial velocity is the superficial velocity adjusted for the cross-section of the actual fluid flow in a packed column. It is related to the superficial velocity through the external porosityof the column, $\varepsilon_{0}$, as follows:

$$
\mu_{i}=\frac{\mu_{s}}{\varepsilon_{0}}
$$

where $\mu_{i}=$ average fluid interstitial linear velocity.

If interstitial velocity is to be substituted for superficial velocity in the Kozeny-Blake equation, one must also include the external porosity term. This term is the compensation factor that maintains the integrity of the value of the permeability coefficient $P_{0}$ between pressure gradient and fluid superficial velocity. The equation then becomes

$$
\frac{\Delta P}{L}=\frac{P_{0}\left(1-\varepsilon_{0}\right)^{2} \mu_{i} \eta}{\varepsilon_{0}^{2} d_{p}^{2}}
$$

3.3. Mobile Phase Velocity. Guiochon does not use either the superficial velocity or the interstitial velocity in the equations in his textbook. Instead, he uses the "mobile phase velocity." Mobile phase velocity is uniquely a chromatographic term. It is the velocity calculated as a result of injecting an unretained, fully permeating solute into the column and measuring the time ofits elution. It is defined as follows:

$$
\mu_{t}=\frac{L}{t_{0}},
$$

where $t_{0}=$ the time it takes an unretained, fully permeating solute to traverse the column and $\mu_{t}=$ mobile phase velocity.
The velocity experienced by the flowing fluid in a column, under steady state conditions, is not altered by the existence of "blind" pores in the particles of that column. Blind pores are pores within the particles which have only one fluid opening; that is they are not through-pores. Accordingly, under steady state conditions, the pores are already filled with identical stagnant fluid and there exists no driving force to exchange this stagnant fluid with the fluid flowing in the interstices of the particles. It will be appreciated that column permeability data is usually taken under steady state conditions in order to take advantage of a constant value for fluid viscosity. Although a solute which is dissolved in the flowing fluid will penetrate the stagnant fluid in the pores (assuming it is not strictly excluded by either physical dimension or chemical interaction) because of molecular diffusion, the driving force for this solute migration is the concentration gradient of the solute between the fluid outside the particles and the fluid inside the particles. This intraparticle migration of the solute does not contribute to friction and thus must not be included in velocity considerations related to pressure gradient. Mobile phase velocity, therefore, is not strictly a fluid velocity term; instead, it is a misnomer and it would better be termed the "unretained fully permeating solute velocity."

Although Guiochon defines the relationship between fluid flow and pressure gradient in the context of a residual permeability coefficient, $k_{0}$, and acknowledges that column porosity plays a role in that relationship, his use of mobile phase velocity fails to recognize that molecular diffusion in blind pores of the particle fraction of a column packed with porous particles does not influence pressure gradient.

Mobile phase velocity is related to fluid superficial velocity, however, through the total porosity of the column. "Total column porosity," $\varepsilon_{t}$, is a term peculiar to the chromatographic literature which relates to the use of columns packed with porous particles. It is the fraction of the column volume taken up by the free space between the particles plus the fraction of the column volume taken up by the free space in the internal pores of the particles. Thus, it is the sum of the external and the internal column porosities:

$$
\varepsilon_{t}=\varepsilon_{0}+\varepsilon_{i}
$$

where $\varepsilon_{t}=$ total column porosity and $\varepsilon_{i}=$ internal column porosity.

Mobile phase velocity is related to fluid superficial velocity through the total porosity of the column as follows:

$$
\mu_{t} \varepsilon_{t}=\mu_{s}
$$

Thus, if mobile phase velocity is used in the Kozeny-Blake equation, one must insert the compensation factor $\varepsilon_{t}$ into the equation in order to maintain the integrity of the value of the permeability coefficient, $P_{0}$, between pressure gradient and fluid superficial velocity, and the equation then becomes

$$
\frac{\Delta P}{L}=\frac{P_{0}\left(1-\varepsilon_{0}\right)^{2} \mu_{t} \varepsilon_{t} \eta}{\varepsilon_{0}^{3} d_{p}^{2}} .
$$


And, now reconciling (1) and (3), we get

$$
\frac{P_{0}\left(1-\varepsilon_{0}\right)^{2} \mu_{s}}{\varepsilon_{0}^{3} d_{p}^{2}}=\frac{\mu_{t} \varepsilon_{t}}{k_{0}\left(d_{p m} \Omega_{p}\right)^{2}} .
$$

\section{The Flow Resistance Parameter}

Before proceeding any further, we digress to define another term which will become important to our understanding of Guiochon's work. The chromatographic literature contains a term called the "flow resistance parameter" [7]. The fluid velocity underlying this parameter is based upon Giddings' definition of "mean fluid velocity through a cross-section" and is found in his 1965 text book at page $207[8]^{2}$. It is defined as follows:

$$
\phi=\frac{\Delta P d_{p}^{2}}{\mu_{t} \eta L},
$$

where $\phi=$ flow resistance parameter.

The flow resistance parameter, therefore, is equivalent to the reciprocal of Guiochon's term $k_{0}$ :

$$
\phi=\frac{1}{k_{0}} \text {. }
$$

\section{Refining the Terms For Column Porosity}

In order to continue with our comparison of Guiochon's teaching to that embodied in the Kozeny-Blake equation and, in particular, to accommodate columns packed with porous particles, we need to refine the porosity dependence term in the Kozeny-Blake equation. To accomplish this, we adopt the definition of column porosity as taught by J. Calvin Giddings [8]; namely,

$$
\Psi=\frac{\left(1-\varepsilon_{t} \Phi\right)^{2}}{\left(\varepsilon_{t} \Phi\right)^{3}}
$$

where $\Psi=$ the porosity dependence term in Kozeny-Blake (porous particles) and

$$
\Phi=\frac{\varepsilon_{0}}{\varepsilon_{t}},
$$

where $\Phi=$ the ratio between the external and total column porosities.

Using this methodology in the most general case involving porous particles to express total column porosity (where total column porosity is not the same as external column porosity) as opposed to in the special case involving nonporous particles (where total column porosity and external column porosity are the same), the Kozeny-Blake equation (using mobile phase velocity as in (10)) may now be reexpressed as follows:

$$
\frac{\Delta P}{L}=\frac{P_{0}\left(1-\varepsilon_{t} \Phi\right)^{2} \mu_{t} \varepsilon_{t} \eta}{\left(\varepsilon_{t} \Phi\right)^{3}\left(d_{p}\right)^{2}}
$$

It will be appreciated that in the special case of columns packed with nonporous particles, (17) is identical to (3) because here $\varepsilon_{t}=\varepsilon_{0}$ and, thus, $\mu_{t}=\mu_{i}=\mu_{s} / \varepsilon_{0}$ and $\Phi=1$.

\section{The Relationship between $k_{0}$ and $P_{0}$}

We can now connect the teaching of Guiochon to that embodied in the Kozeny-Blake equation as follows:

$$
P_{0}=\frac{1}{k_{0} \Psi}
$$

or

$$
P_{0}=\frac{\phi}{\Psi}
$$

As illustrated by (19), the term $P_{0}$ in the Kozeny-Blake equation is the flow resistance parameter normalized for the column porosity dependence term.

Note that if $P_{0}$ is a true constant, the two parameters upon which it depends, $\phi$ and $\Psi$, will vary in direct proportion to each other. This is illustrated by the data in Table 1 herein, which is a reference chart where particle porosity, $\varepsilon_{p}$, is varied from nonporous at one extreme to highly porous at the other, where $\varepsilon_{p}=$ volume of the free space within the particles in a packed column/the total volume of all the particles in the column.

Figure 1(a) is a plot of the data contained in Table 1 as $\phi$ versus $\Psi$. Proceeding from the lower left hand corner to the upper right hand corner of the plot, particle porosity increases. It is obvious from the plot that all the data in Table 1 falls on a single line with a slope of 267 , which we say is the constant value of $P_{0}$. As is illustrated by the plot, in the range of porosity covered by the data, the values for $\phi$ vary from about 500 to about 1900 and, correspondingly, the values of $\Psi$ vary from approximately 2 to approximately 7 . Note also that the upper boundary value of $\Psi$ for packed columns is about 5.5 with larger values related to capillaries.

Finally, we point out that once the value of $P_{0}$ is correctly identified, a measured value for the flow resistance parameter may then be used to calculate the value of the external porosity of the column, $\varepsilon_{0}$, when the latter's value has not or cannot be measured.

\section{Guiochon's Modified Permeability Coefficient, $P_{t}$}

As reflected in some of his recent publications, which we will explore in more depth later, Guiochon inadvertently, apparently, confuses the term $P_{0}$ with another parameter, $P_{t}$, which we identify here and define for the first time. $P_{t}$ is not the same as $P_{0}$ because it is based upon Guiochon's unique (from a permeability perspective) teaching for fluid velocity. It is defined as follows:

$$
P_{t}=P_{0} \varepsilon_{t}
$$

where $P_{t}=$ Guiochon's modified permeability coefficient.

It will be appreciated that Guiochon's $P_{t}$, if used in the Kozeny-Blake equation in place of $P_{0}$, accommodates his use of the mobile phase velocity in a way that is different from, but equivalent to, a direct conversion of mobile phase velocity to superficial velocity. 


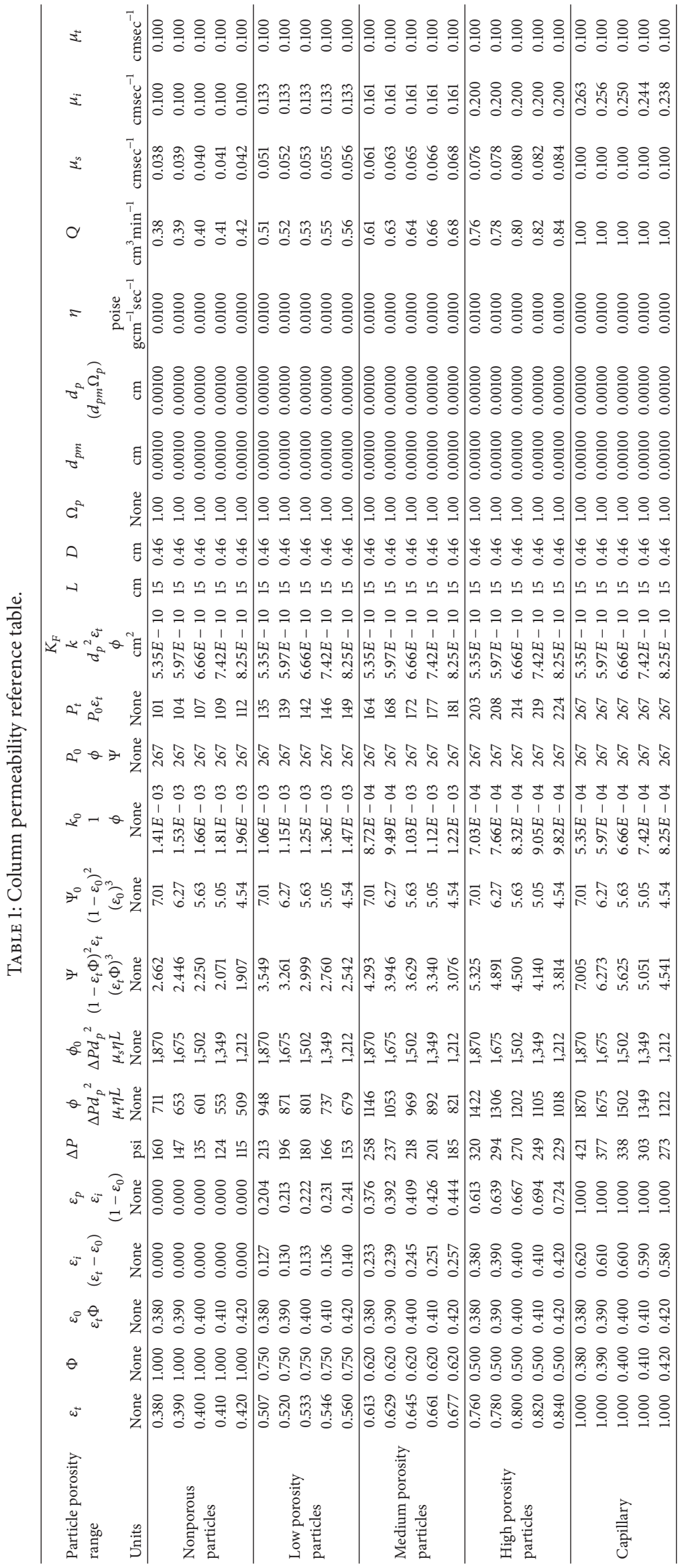




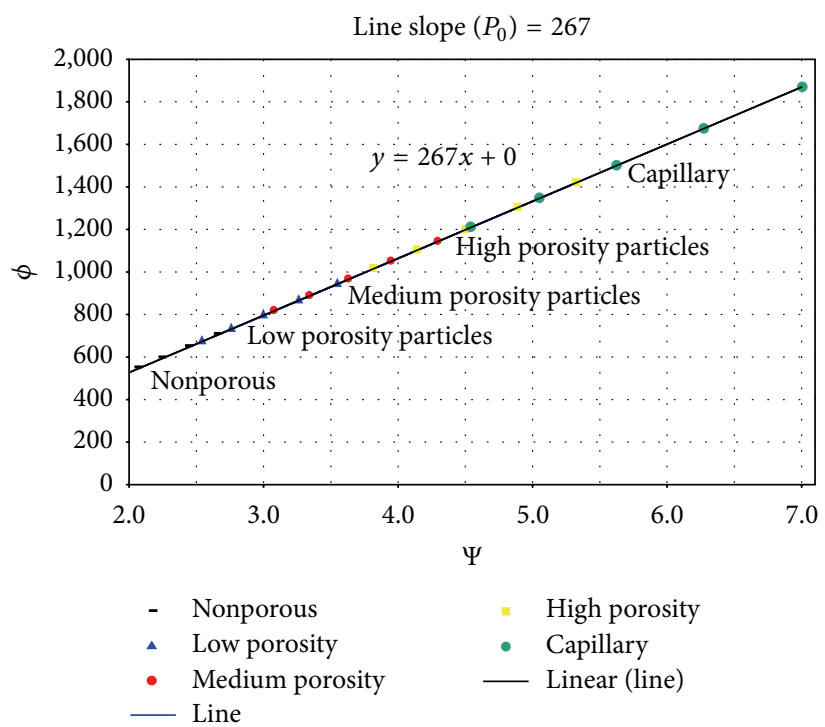

(a)

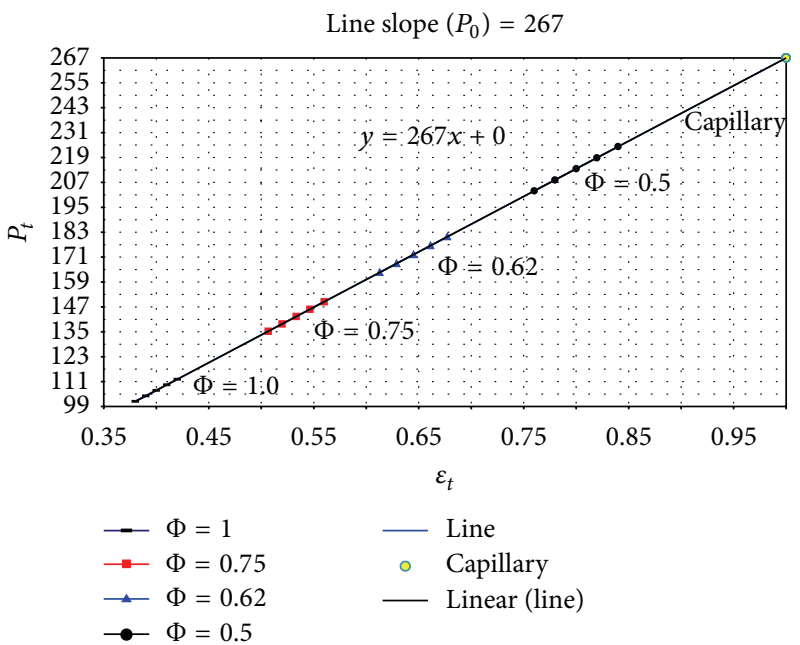

(b)

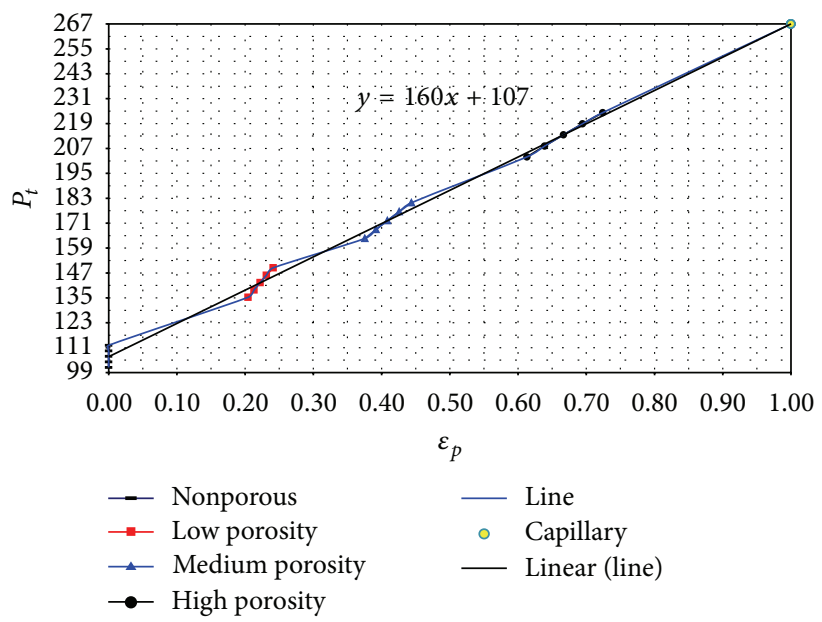

(c)

Figure 1: (a) The balance between $\phi$ and $\Psi$. (b) Column permeability reference chart. (c) Particle permeability reference chart.

In the 2006 edition of their textbook [9] Guiochon and his coauthors provide the following worked example of his pressure drop/fluid flow teaching. ${ }^{3}$

In Table 2, a cross-reference is established between Guiochon's teaching for columns packed with both porous and nonporous particles, the Kozeny-Blake equation (as modified by Carman), and, for validation purposes, Giddings' teaching based upon actual permeability measurements from his Table 5.3-1 in his 1965 text [8]. ${ }^{4}$

In his worked example, Guiochon's fluid velocity, $u$, is found in his text as his equation (2.56) [9], at page 61 , and equates to mobile phase, not superficial, velocity. Accordingly, when applied to columns packed with porous particles, his worked example combines the contribution to fluid velocity of mass transfer by molecular diffusion in the free space within the particles with mass transfer by convection in the free space between the particles. As discussed above, if one is attempting to compare Guiochon's teaching for the value of the permeability coefficient with that of the Kozeny-Blake equation, the conversion factor is the total porosity of the column.

In addition, because Guiochon's particle size parameter has an embedded assumption due to particle shape/roughness, his worked example also commingles the contribution to spherical particle diameter equivalent of particle shape/roughness with other nonparticle variables. Unfortunately, his worked example is not based upon completely spherical particles where this would not be an issue. We know this because he states that $k_{0}$ in his worked example equals $1 \times 10^{-3}$, which he says is the value of the permeability coefficient for columns packed with irregular particles.

In order to move forward, one must simplify. If this were to be done experimentally, it would best be accomplished by experiments with nonporous particles, in which case the variable of molecular diffusion in the pores of the particles 


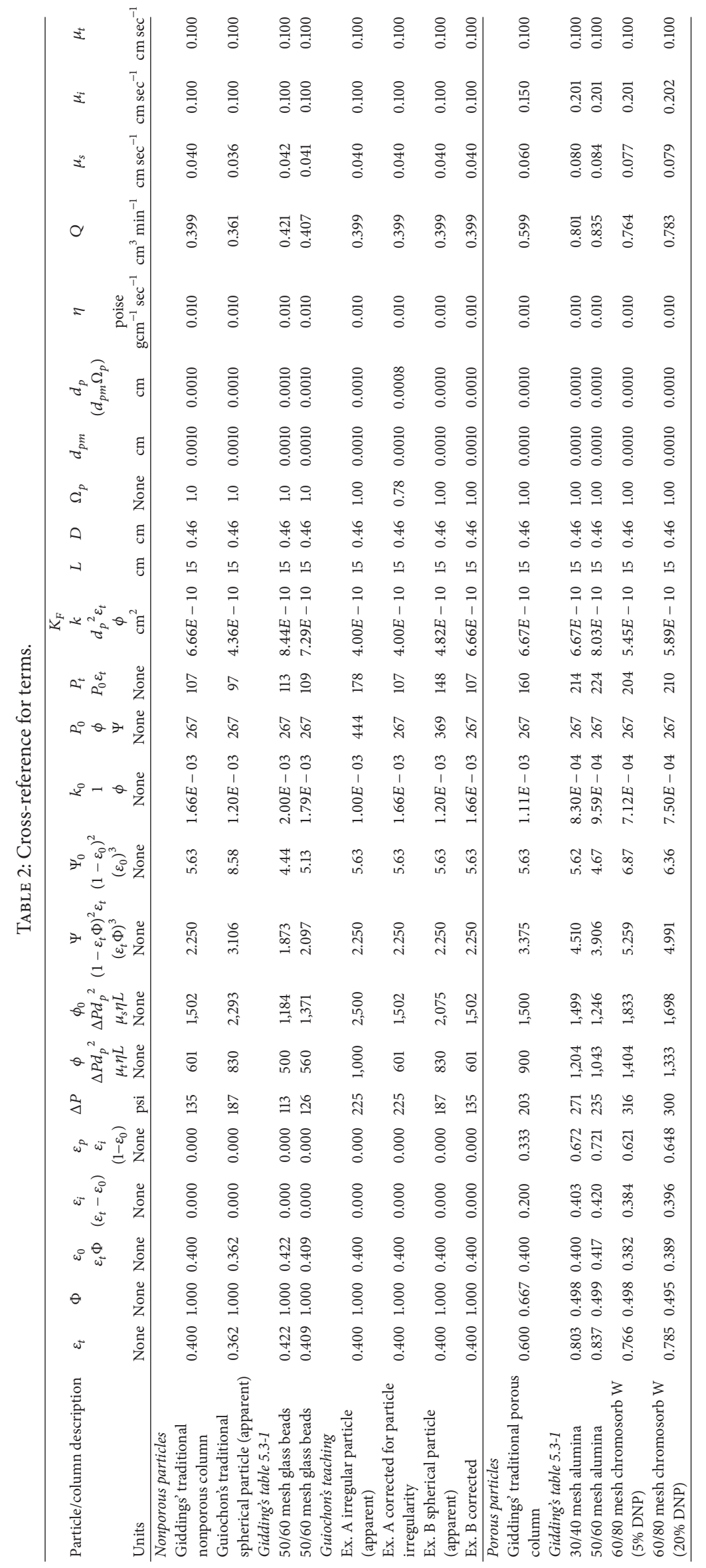


would be eliminated and by using only smooth, spherical particles, such as glass beads, in which case the variable of particle shape/roughness would be eliminated. If both of these things were done, one would be able to directly and unambiguously determine the true value of $P_{0}{ }^{5}$

Accordingly, since his textbook contains a general teaching without any expressed restrictions with respect to particle porosity, let us first assume that the column in Guiochon's worked example is packed with nonporous particles. Secondly, let us assume that the value for the external porosity of the column (which is the same as the total porosity in a column packed with nonporous particles) is the value traditionally assumed for a typical, well-packed column, that is, 0.4 .

As illustrated by example A in Table 2, this would lead to an apparent value for $P_{0}$ of 444 , which is obviously much too high. ${ }^{6}$ Using the adjusted particle size of 8 micrometers (rounded), which is calculated based on a value of 0.78 for $\Omega_{p}$, we derive values of 267 for $P_{0}$ and 107 for $P_{t}$ (the relationship between these two values is, of course, equal to the total porosity of the column, $\varepsilon_{t}$, as is dictated by (20)).

Similarly, as illustrated by example B in Table 2 , when the column of Guiochon's worked example is adapted to accommodate spherical, nonporous particles, Guiochon's teaching of the value for $k_{0}$ of $1.2 \times 10^{-3}$ results in an apparent value of 369 for $P_{0}{ }^{7}$, again, too high $[10,11]$. On the other hand, if we correct the value of $P_{0}$, we get a column external porosity of 0.3620 ,which is too low for a typical well-packed column $\left(\varepsilon_{0}=0.4\right)$ [8]. Moreover, in the case of both corrections, Guiochon's teaching overstates the pressure gradient (187 psi compared to the 135 psi which Giddings' experiments would generate). Accordingly, it is only when we correct for the pressure drop that we achieve appropriate values of 0.4 for $\varepsilon_{0}, 267$ for $P_{0}$, and 107 for $P_{t}$.

As is evident from Table 2, when the porosity of columns packed with nonporous particles varies, the value of $P_{t}$ also varies because it is based upon the measurement of mobile phase velocity, which, in turn, changes with the porosity of the column. Indeed, for typical columns packed with nonporous particles having external porosities close to $0.4(0.38-0.42)$ [8], Table 2 reveals that the value of $P_{t}$ varies by as much as 6 points, while the value of $P_{0}$ remains constant at 267.

Having established a range of values for $P_{t}$ based upon columns packed with nonporous particles, one can now proceed to evaluate the range of values for $P_{t}$ based upon columns packed with porous particles, which is to say that we can now evaluate the impact of particle porosity on permeability. Among other things, Table 2 shows that when it comes to porous particles, Guiochon's $P_{t}$ is even more variable (and, thus, even less useful) because the value of the coefficient can differ on a column-to-column basis by as much as 90 points, depending on the combination of the internal andexternal column porosities at play.

Figures 1(a) and 1(b) are a plot of the data found in Table 1. In this plot, Guiochon's modified permeability coefficient, $P_{t}$, is plotted against column total porosity, $\varepsilon_{t}$. Once again, it will be appreciated that (a) all data falls on a line with a slope of 267 which represents the value of $P_{0}$ and (b) the line has no intercept, which means that when $\varepsilon_{t}$ is zero, $P_{t}$ is also zero. As shown in the plot, values of the parameter $\Phi$ are highest at low values of $P_{t}$ and lowest at high values of $P_{t}$. Further, as is evidenced by the respective definitions for the terms $P_{t}$ and $P_{0}$ contained herein, the only time that the value of Guiochon's $P_{t}$ does not differ from the value of $P_{0}(267)$ is when the value of $\varepsilon_{t}$ is 1 , its value in a theoretical packed column devoid of particles, that is, acapillary.

Finally, Figure 1(c) is another plot of the data in Table 1, but this time the values for $P_{t}$ are plotted against particle porosity, $\varepsilon_{p}$. The equation of this line does have an intercept, which represents the value of $P_{t}$ when the particles are nonporous. It is also obvious from the equation of this line that it is only when particle porosity is unity, that is, in a capillary, that the value of $P_{t}$ is 267 .

It will be appreciated that since $\varepsilon_{t}$ is the sum of the internal and external porosities of a packed column, any given value for $P_{t}$ involving a column packed with porous particles can represent many different combinations of these parameters. Vice versa, any given value for $\varepsilon_{t}$ can generate many different values for $P_{t}$ depending on the amount of particle porosity which is present. On the other hand, as is pointed out earlier, column internal porosity and/or particle porosity play no role in determining the permeability of a packed column; the only porosity parameter which is relevant for such purposes is column external porosity. In other words, as a permeability coefficient, $P_{t}$ is not a particularly informative concept because it still fails to provide any meaningful tie to the underlying physical phenomena that govern the pressure drop/fluid flow relationship.

In the case of permeability measurements, one quantifies only total pressure drop. Pressure drop, however, is a commingling of the contributions of the several different parameters embodied in the pressure/flow relationship. Therefore, one must accurately represent the contribution of each factor before adding them together to equal the pressure drop. Obviously, if one mistakes the contribution of any given element, for instance particle size, this will skew the contribution of some other element when summed, for instance, column porosity.

Our frame of reference has been calibrated with respect to the interrelationships between the values of $P_{0}, P_{t}, \varepsilon_{t}$, and $\varepsilon_{p}$ based on the data reported in Giddings' table 5.3-1 in his 1965 textbook. Since his data base was derived from experiments using nonporous particles, the permeability results of which were extended to columns packed with fully porous particles by using his $\Phi$ parameter, the validity of which, in turn, was established by independent means, there is no uncertainty with respect to the critical variable of external porosity, $\varepsilon_{0}$, inherent in our frame of reference. It is for this reason, therefore, that our frame of reference trumps any reported data which uses any other technique, including the technique of inverse size exclusion chromatography by itself to establish values for $\varepsilon_{0}$ [12]. In addition, our frame of reference is internally consistent due to the grounding of the parameters at the boundary condition of a theoretical capillary, at which point the values of $\varepsilon_{t}$ and $\varepsilon_{p}$ are unity and the values of $P_{0}$ and $P_{t}$ are equal. In essence, these interrelationships exemplify the conservation laws of nature which are the ultimate standard 
of measure when considering partial fractions of a single entity.

Consequently, as Figures 1(b) and 1(c) illustrate, if one knows based upon other independent means, the value of a column's total porosity or its particles' porosity, respectively, one can at least verify whether results of permeability experiments expressed in terms of $P_{t}$ are reasonable. Or, to put it another way, if the $P_{t}$ results reported do not conform to what these plots tell us one should expect from columns and particles based upon independently derived porosity values for the particles under study, we know that something is wrong. Accordingly, this is the way in which we use $P_{t}$ below to analyze and evaluate Guiochon's experimental work of the last decade.

\section{The Origin of Guiochon's Value for Column Permeability}

In 1966, Guiochon wrote a review paper entitled "Problems Raised by the Operation of Gas Chromatographic Columns" [13]. In that paper, he reviewed the development of the permeability equation from Darcy to the then present. Among other things, he reported that "a mean $\varepsilon$ of 0.38 is found for a number of packings obtained from powdered products of various origins, consisting of spherical or irregular particles.... Almost all the measured values are between 0.35 and 0.45 " (p. 14). He then went on to discuss what he called the "semiempirical Blake-Kozeny equation," which he reported as $k=\left[d_{p}^{2} \varepsilon^{2}\right] /\left[150(1-\varepsilon)^{2}\right][13$, p. 15. Equation (11)]. What sticks out, of course, is the fact that Guiochon states here that $P_{0}$ equals 150 , the value for the permeability coefficient championed by Ergun, not the value posited by Carman. To be fair, we should point out that Guiochon gave warning that he was uncomfortable with any particular value for the permeability coefficient. For even though he recited the Kozeny-Blake equation with a value of 150 for its permeability coefficient, he stated that "the values of $k$ given by (this) equation are not very accurate, and the deviations can be as large as 50\%" [13, p. 15]. Guiochon attributed most of this uncertainty to the inability of practitioners of that era to effectively measure the value of a column's external porosity. Since, as he pointed out, "(the Kozeny-Blake) equation is very sensitive to variations in $\varepsilon$," he concluded that the equation is "of little use" and that it was better practice to rely upon grosser measurements of permeability [13, p. 15]. Thus, Guiochon at this timeframe made a conscious decision to stay within the relatively safe, but admittedly more obscure, boundaries of Darcy's teaching on column permeability.

In 2006, however, Guiochon emerged from under the shadow of Darcy permeability when he published a review paper which purported to be a comprehensive summary of the current state of the art in chromatography [14]. In light of the fact that Guiochon and his coauthors had virtually contemporaneously published a new version of their textbook, one would have expected that Guiochon would take this opportunity to reaffirm his textbook teaching. So, it should come as no surprise that he would open his discussion of the pressure drop/fluid flow relationship with the following assertion: "the permeability of packed beds used in liquid chromatography is in the order of $1 \times 10^{-3}$ ” [14, p. 9]. As one would expect, the authority which Guiochon cites for this seemingly familiar proposition is the 2006 edition of his own textbook. For the first time, however, he also provides some citations to third party sources. In particular, he cites a 1997 chromatography handbook written by Uwe Neue, Chief Scientist for Waters Corporation [15, p. 9]. As one can see, Neue's equation-like Guiochon's-does indeed postulate a permeability coefficient with a value of $1 \times 10^{-3}$.

At this point, however, Guiochon abandons his traditional caution about the value of the permeability coefficient and marries his permeability equation to the KozenyBlake equation, thereby endorsing not only the porosity dependence term in that equation but also a specific value for $P_{0}$. Referring to his value of $1 \times 10^{-3}$, he states the following: "the specific column permeability is related to the column porosity through the Kozeny-Carman equation: $k_{f}=$

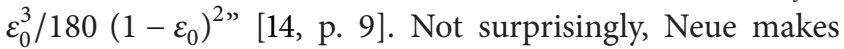
the same connection: "the specific permeability depends on the particle size $d_{p}$ and the interstitial porosity $\varepsilon_{0}$ of the packed bed. The relationship is known as the Kozeny-Carman equation: $B_{0}=(1 / 185)\left(\varepsilon_{0}^{3} d_{p}^{2} /\left(1-\varepsilon_{0}\right)^{2}\right)$ " [15, p. 30].

Neue's permeability equation and Guiochon's textbook permeability equation, however, differ in that Neue uses the superficial, not the mobile phase, velocity. Accordingly, since all other features of their equations are the same, one would expect them to get different values for their permeability coefficients. Neue himself recognizes this when he says in his handbook "this value [1000] is also in good agreement with our experience, but values as low as 500 have been reported in the literature. ${ }^{8}$ However, there are several reasons why different values reported by different workers do not represent true differences in the resistance parameter. First, lower values are obtained for porous packings if the specific permeability is measured on the basis of the breakthrough time of an unretained peak instead of using the flow rate"; see $[15$, p. 32] (when he distinguishes the "breakthrough time of an unretained peak" from the "flow rate," Neue is, of course, referring to the mobile phase velocity and the superficial velocity, resp.). Consequently, if one takes Neue's point and turns it on its head, one can see that if he and Guiochon were to get the same value for their permeability coefficients [1000], but one (Guiochon) is using mobile phase velocity to derive it and the other (Neue) uses superficial velocity to derive it; this would indeed represent a "true difference" in their equations.

So, how can Guiochon cite both Neue and himself for the proposition in his review paper that "the permeability of the packed beds used in liquid chromatography is in the order of $1 \times 10^{-3}$ "? The answer is that, at least for purposes of his review paper, he adopts Kozeny-Blake's fluid velocity convention in place of the velocity frame he uses in his textbook. Here is what he says: "this approximation $\left[1 \times 10^{-3}\right]$ is valid only if the superficial velocity is used" [14, p. 9] (emphasis supplied).

As a matter of pure mathematics, Guiochon's permeability coefficient of $1 \times 10^{-3}$ can only be equal to $\varepsilon_{0}^{3} / 180\left(1-\varepsilon_{0}\right)^{2}$ if the value of $\varepsilon_{0}$ is 0.4 , the typical value for the interstitial 
fraction of a well-packed column. Guiochon confirms in his review paper that this is indeed what he assumes in his statement of the Kozeny-Blake equation [14, p. 9]. Accordingly, his adoption of the value of 180 for $P_{0}$ solely depends on the validity of his assertion that the value of the permeability coefficient is $1 \times 10^{-3}$.

We already know from our discussion of Guiochon's textbook teaching that when this value is associated with a permeability equation based on mobile phase velocity, it is wrong. As reflected in our Table 2, when Guiochon's worked example involving this figure is corrected to account for the embedded factor due to the irregularity of the particles which his worked example assumes, the value of the permeability coefficient generated by his equation is not $1 \times 10^{-3}$; it is 1.66 $\times 10^{-3}$.

So, where did this value of $1 \times 10^{-3}$ come from? Although Guiochon in his review paper cites Neue's handbook for this value, Neue himself offers absolutely no authority for it. On the other hand, Guiochon does provide one other citation in his review paper for this value. The citation is to an article by Halász et al. published in 1975 [16].

If one then goes to that article, one sees that Halász et al. do indeed proffer the same equation as Neue (albeit in a somewhat different format) and, yes, it does contain the value of $1 \times 10^{-3}$. See [16, Equation (1)]. Nevertheless, the authors fail to identify from whence they obtained this value. On the other hand, they do make reference to a paper that Endele et al. wrote a year earlier, in 1974, with Klaus Unger entitled "Influence of the Particle Size (5-35 $\mu$ ) of Spherical Silica on Column Efficiencies in High-Pressure Liquid Chromatography" [17]. It appears that this is where the body is buried; for it is in this article that Halász reports having actually derived a value of $1 \times 10^{-3}$ for the permeability coefficient.

In summarizing the results of their experiments, which, incidentally, are based on measurements of superficial, not mobile phase velocity (see their Equation (7)), Halász et al. calculate an average value for the permeability coefficient (which they notate as $K_{F}$ ) of $d_{p}^{2} / 1080$. Accordingly, they state the following: "[I]t is proposed to define an average particle size, $d_{p}$, for columns packed with spherical silica using the balanced density packing method by the equation $d_{p}^{2}=$ $K_{F} / 1000=F \eta L / 10^{3} r^{2} \pi \Delta P^{\prime \prime}[17$, p. 382 their Equation (7)].

To recapitulate, it thus appears that (1) Halász was the source of the value of $1 \times 10^{-3}$ for the permeability coefficient which Guiochon adopts in his review paper, (2) Halász' value was based upon measurements which involved spherical particles, and (3) Halász' value was properly derived from an equation which utilized the superficial velocity as the fluid velocity.

However, we still have a problem: if we are going to accept the value of $1 \times 10^{-3}$ as a basis for calculating the constant in Kozeny-Blake, we still need to know the value of Halász' columns' external porosity. For example, in the absence of measured values for the external porosity, Guiochon's assertion in his review paper that the value of the constant is 180 is without foundation.
Unfortunately, however, Halász bases his methodology on "assumed" versus "measured" values for external porosity. Nevertheless, he does provide some clues that may help us peel back this onion. First, he says that his value for the permeability coefficient applies to "columns using the balanced density packing method." Secondly, after stating his proposed value for the coefficient, we find the following telltale sentence: "the permeability of columns packed by the conventional "dry" method are worse by a factor of about 2 than those packed by the balanced density method" [17, p. 382]. For this proposition, Halász cites yet another of his own articles, this time a paper written by himself and M. Naefe in 1972 entitled "Influence of Column Parameters on Peak Broadening in High-Pressure Liquid Chromatography" [18].

When one follows this thread by going to Halász's 1972 paper, one finds a possible solution to the puzzle. For this is where Halász describes his calculations of the permeability coefficient from experiments with a number of conventional, dry-packed columns containing spherical silica particles. Not surprisingly, the resulting value for the permeability coefficient for such columns was not $1 \times 10^{-3}$. Instead, Halász says that his experiments with such columns resulted in the following value for the permeability coefficient: " $K \sim$ $d_{p}^{2} / 2000$ " [18, p. 78]. In other words, consistent with what Halász says in his 1974 article, the value for the coefficient for these dry-packed columns was indeed "worse by a factor of about 2" compared to its value for columns prepared with the balanced density method.

The obvious implication of this is that when Halász got a value of $1 \times 10^{-3}$ for the permeability coefficient in 1974, he must have been evaluating columns that were packed to an interstitial fraction considerably higher than the external porosity of the columns that were the subject of his 1972 experiments. Our problem, however, is still not resolved because Halász did not accurately determine the external porosity of the columns under study in either the 1972 or 1974 columns.

We surmise that, in their 1972 paper, Halász and his coauthors, made use of the teaching of Giddings outlined in his 1965 textbook, which at the time of their 1972 paper was seven years old. Here is what they say: "experience has shown that in a regular packed column, with the sieve fractions usual for chromatography, $\varepsilon_{0}$ is independent of the particle size if the particles are more or less spherical. In an acceptable approximation, $\varepsilon_{0}$ is equal to 0.4 . In those columns packed with nonporous supports $u_{v}$ and $u$ are identical. Using porous supports (i.e., silica gel, alumina, chromosorb, Porasil, and so forth), $u=4 F /\left(\pi d_{c}^{2} \varepsilon_{T}\right)$, where $\varepsilon_{T}$ is the total porosity and indicates the pore volume of the support." It will be appreciated that $u_{v}$ stands for interstitial velocity (our $\mu_{i}$ ) and $u$ stands for mobile phase velocity (our $\mu_{t}$ ). The "experience" which Halász and his coauthors are referring to concerning "columns packed with nonporous supports, alumina, and chromosorb" is that recorded by Giddings in Table 5.3-1 in his 1965 text. Continuing to establish the interrelationships inherent in the various entities involved in chromatographic columns with respect to both interstitial and mobile phase velocity, the authors conclude: "formally $u_{v}$ 
can be calculated for a porous support, assuming $\varepsilon_{0}$ is equal to 0.4." (emphasis supplied). The authors then announce the methodology underlying their frame of reference with regard to column permeability as follows: "we determined in all of our columns the $u / u_{v}$, ratio to be 0.56 with a differential refractometer. Consequently, the total porosity $\varepsilon_{t}$ was equal to 0.714 ." It will be appreciated that their ratio of $u / u_{v}$ is one and the same as Giddings' $\Phi$ parameter, as utilized by him in his 1965 textbook and as we define hereinabove.

We can now identify the origin of the discrepancy between Halász and Giddings and, by extension, between Halász and Guiochon (and Neue). For even though Halász makes use of the results in Giddings' Table 5.3-1, he fails to appreciate the teaching inherent therein regarding the importance of an accurate value for column external porosity when using porous particles. In establishing his values for $\Phi$, Giddings was careful to ground his values for the external porosity of columns packed with porous particles in a comparison of their measured pressure drops with measured pressure drops in columns packed with nonporous particles, where the values for (external) porosity were accurate because they are equal to the columns' total porosity.

In their 1972 paper, Halász et al. used a refractometer to detect the inert solute, $n$-pentane, in the mobile phase of $n$ heptane. They injected the $n$-pentane into the flowing mobile phase, the flow rate of which they presumably measured with a volumetric cylinder and stop watch. Since the exit time of the $n$-pentane peak was identified by the refractometer, their measurement of holdup volume was accurate and, accordingly, so was their value of 0.714 for $\varepsilon_{t}$ (there is no uncertainty related to column total porosity when using inert solutes). This, in turn, led to an accurate value for their $u$ term, mobile phase velocity. However, since they did not measure the interstitial velocity, $u_{v}$, but rather used an estimate based upon the measured flow rate and an "assumed" value of 0.4 for external porosity, their calculated/estimated value for $u_{v}$ was arbitrary. This, in turn, means that the value of 0.56 for their $\Phi$ ratio was likewise arbitrary.

In Table 3 herein, we show the results for column number 1 in the 1972 study which we designate as Column $A_{1}$. Note that the authors' raw values correspond to a value of 500 for $P_{0}$, a value which is obviously far too high. As is plainly evident in our Figures 2 and 3, this column data set does not conform in any reasonable fashion to the norms in our reference charts. Accordingly, the data is badly flawed. In our corrected data for Column $A_{1}$ in Table 3 herein, we adjust the column external porosity to the lowest value permitted in our frame of reference (0.38). This correction results in a revised value for Giddings' $\Phi$ parameter of 0.532 . In addition, we are compelled to adjust the particle downward (to 11 micrometers, approx.), as is also dictated by our frame of reference. We justify our low corrected value for external porosity and our downward adjustment for particle size on the aggressive "dry-packing" process described by the authors; "[T] the best results were achieved with the following method: the column (i.d. = $2 \mathrm{~mm}$ ), $25 \mathrm{~cm}$ long, was packed with 25 equal portions of the "brush." After adding each portion, the column was vibrated and afterward tapped on the floor and also vigorously tapped with a rod from the side." Based upon a column packing experience of the principal author herein spanning more than 30 years, we believe that these packing conditions generated a packed column with a low external porosity and also one in which the particles experienced significant breakage.

The main differences between the columns in the 1972 and 1974 papers concerned the particle sizes and the methods used to pack the columns. The particle diameters in the 1972 study fell in a range of about 15-200 micrometers while those in the 1974 study were much smaller, being in the range of 540 micrometers approximately. Moreover, while the columns in the 1972 study were dry-packed, the columns in the 1974 study were slurry-packed (what Halász calls "the balanced density packing method"). The authors of the 1974 paper recite that their permeability results shown in their Table 4 correspond to values for $\phi_{0}$ of 1080 and values for $\phi$ of 910 . In Table 3 herein we show the results for the 1974 study as an average value in our column designated as $\mathrm{A}_{2}$. Note that the raw data reported by the authors, which again has the built-in assumption that the column external porosity was 0.4 , places the value of $P_{0}$ at 192. In our corrected values for Column $\mathrm{A}_{2}$, we show that a value of 267 for $P_{0}$ places the column external porosity at a value of 0.43 . The ratio of the value for $\phi$ of 910 , when compared to the value for $\phi$ in the 1972 study of 2007, is about $2.2(2007 / 910=2.2)$, which is in good agreement with the authors' statement that the permeability of the 1972 columns was worse by a factor of about 2 . Again, based on the experience of the principal author herein in the packing of hundreds of thousands of commercially sold slurry packed columns, we conclude that the balanced density technique described by the authors in the 1974 paper is fully capable of producing columns with this high and external porosity.

In summary, we conclude that, for purposes of the Kozeny-Blake equation, (a) the external porosity of the Halász 1972 columns was 0.38 , (b) the external porosity of the columns which he tested in 1974 was 0.43 , and (c) Halász's 1972 and 1974 studies, when properly analyzed, corroborate Giddings' value for $P_{0}$ of 267, not Carman's value of 180 . Although the fault for all of the misdirection about the value of the permeability coefficient being $1 \times 10^{-3}$, therefore, rests primarily on the shoulders of Halász, one has to question why Guiochon (and Neue) so readily adopted it. Whatever may be the reason, Guiochon's support for this proposition has been and continues to be a cause for much of the conventional acceptance of Carman's (erroneous) figure of 180 as the characteristic value of the coefficient in the Kozeny-Blake equation.

\section{Guiochon's Experimental Data}

In addition to the data from Halász 1972 and 1974 studies, Table 3 herein contains data from a representative sample of Guiochon's personal experimental permeability investigations over approximately the last decade, as reported in the numerous trade journals, particularly the Journal of Chromatography $A$. In each case, the table has a single row dedicated to Guiochon's reported raw data and a single row dedicated to corrected values for each column based on the reference chart in Table 1 herein. 


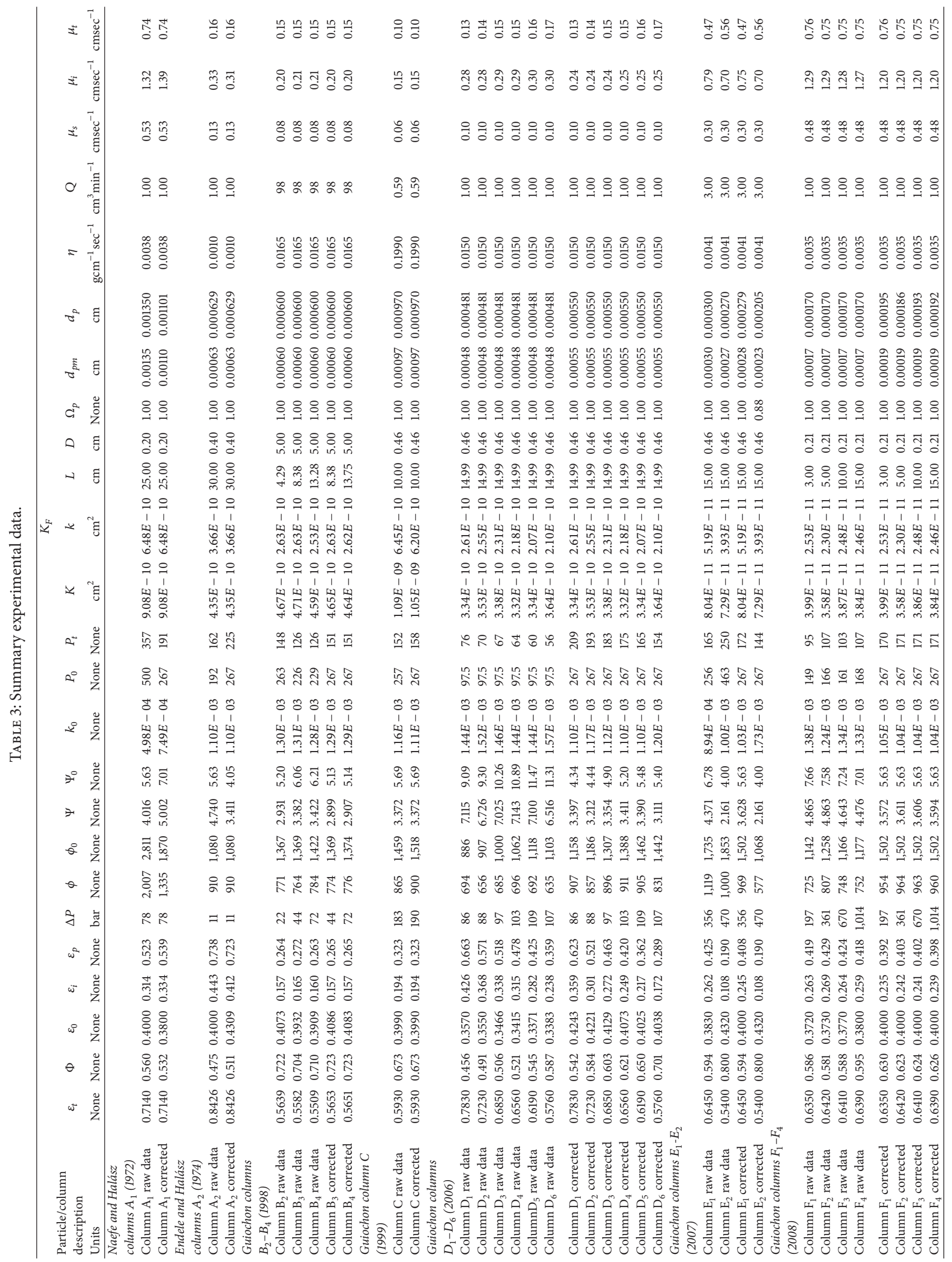




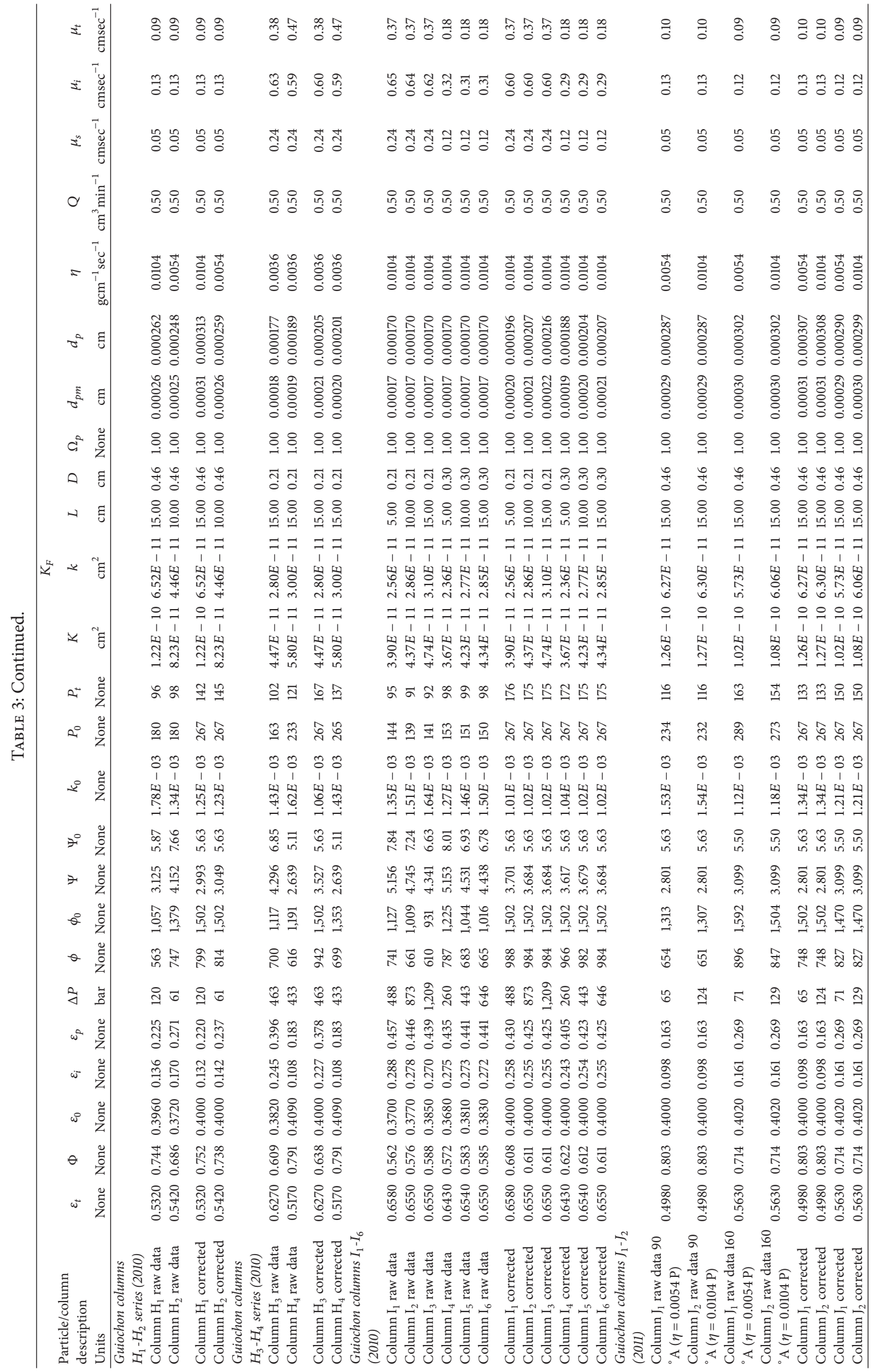




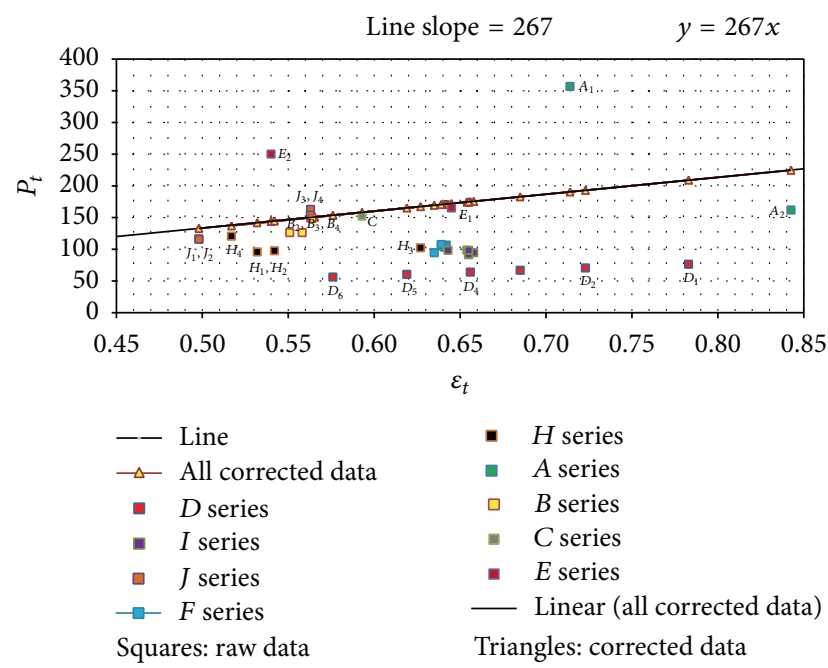

FIGURE 2: Summary of experimental results.

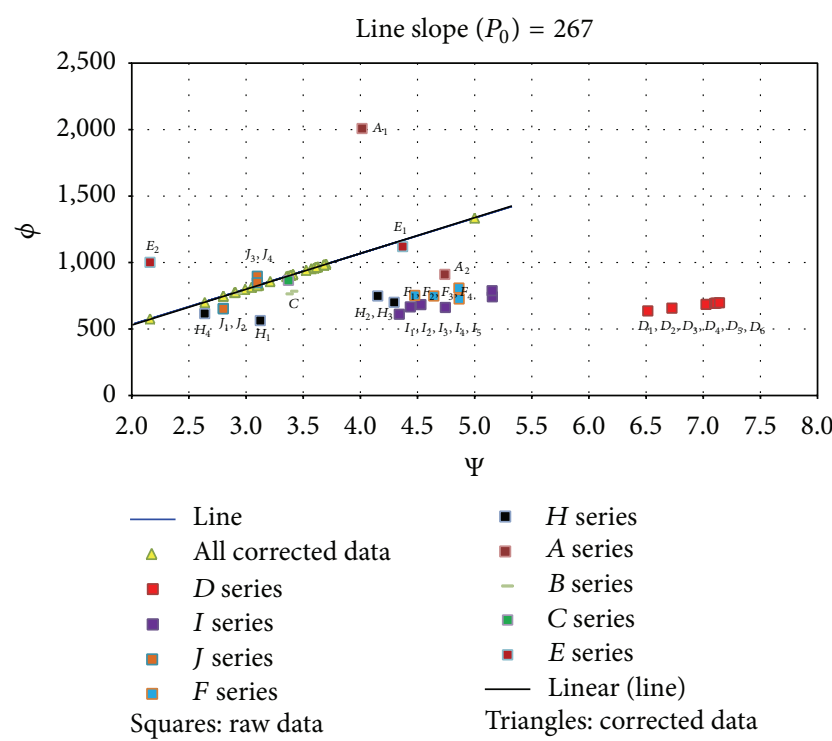

FIGURE 3: Summary of experimental results.

Figures 2 and 3 herein are graphical representations of Table 3/s raw and corrected data for Guiochon's columns plotted in the same reference frames as in Figures 1(a) and 1(b), respectively.

As shown in the plots, of the thirty-one total columns evaluated in this data base, only six columns had reported raw data values for $P_{0}$ close to the norm. These were columns $\mathrm{B}_{2}, \mathrm{C}, \mathrm{E}_{1}, \mathrm{H}_{4}, \mathrm{~J}_{1}$, and $\mathrm{J}_{2}$. Interestingly, of the six conforming columns, 3 of the columns were packed with fully porous particles and 3 columns with partially porous particles. It can be seen, however, that when properly interpreted and adjusted, all of Guiochon's experimental data falls fairly within the norms outlined in the reference chart in Table 1. In order to explore the reasons why the raw data does not conform more closely to the norms outlined in Table 1, each of Guiochon's studies included in Table 3 is evaluated in turn.
Finally, Table 4 herein is a list of symbols and parameter definitions used throughout this paper as well as their units of measure.

9.1. Guiochon's 1998 Study. In a 1998 publication by Koh and Guiochon entitled "Effect of the Column Length on the Characteristics of the Packed Bed and the Column Efficiency in a Dynamic Axial Compression Column" [19], Guiochon and his coauthor report the results of a study they had carried out on column packing using a rather novel technique which was a combination of slurry packing and mechanical compression.

The study involved the packing of a single cylinder of diameter $5 \mathrm{~cm}$ to various column lengths ranging between 1 and $25 \mathrm{~cm}$, approximately. The particles were all silica based and coated with a reverse stationary phase $\mathrm{C}_{18}$. However, two different categories of particles were involved. One category contained highly irregular particles which were about 130 micrometers in average diameter. In addition, these particles had a high specific pore volume $(1.1 \mathrm{~mL} / \mathrm{g})$ and exhibited significant particle breakage under the packing conditions investigated in the study. Conversely, the other particle category involved very small particles (6 micrometers) which were spherical in shape, had a low specific pore volume $(0.3 \mathrm{~mL} / \mathrm{g})$, and exhibited a greater ability to withstand particle breakage under the study's packing conditions.

The permeability data for the irregular particles is neglected herein because, according to the authors, the particles exhibited significant breakage during packing and, without an accurate value for the characteristic dimension of the particle, the data cannot be used in the KozenyBlake model to determine the value of $P_{0}$. The spherical particles, on the other hand, behaved successfully under the packing conditions chosen and yielded values calculated by the authors for $P_{0}$ of $619,268,226$, and 229 for the four different lengths of columns studied. The data for these columns is reported in our Table 3 in the rows for Columns $\mathrm{B}_{1}$ through $\mathrm{B}_{4}$. The higher value of 619 (the shortest column) was rejected by the authors because they felt that either the particles had been somewhat broken during packing or the column frits had been blocked with fines. Accordingly, the permeability data for this column is likewise neglected herein.

The remaining three columns, however, were not consistent with respect to the authors' calculated values for $P_{0}$. Since the particles were identical, the range of values for $P_{0}$ of 226 to 268 must be due to experimental error. The raw data for column $\mathrm{B}_{2}$, which produced a $P_{0}$ value of 268 , is all self-consistent. For example, it generates a value for $P_{t}$ of 148, which is a reasonable value based upon the known low particle porosity for these Nova Pak particles and a corresponding value for $\varepsilon_{0}$ of 0.4073 , which is a reasonable value for a well-packed column. However, the other two columns, columns $\mathrm{B}_{3}$ and $\mathrm{B}_{4}$, had the much lower value of 126 for $P_{t}$. Accordingly, in Table 3 herein, corrected values for column external porosity for columns $\mathrm{B}_{3}$ and $\mathrm{B}_{4}$ are displayed. These corrected values are only slightly different than the reported values, are within the experimental error of the measurement, and, therefore, in combination with the 
TABLE 4: Glossary of terms.

\begin{tabular}{|c|c|c|}
\hline Symbol & Unit dimensions (cgs) & Definition \\
\hline$A$ & $\mathrm{~cm}^{2}$ & Column cross-sectional area \\
\hline$D$ & $\mathrm{~cm}$ & Column diameter \\
\hline$d_{p}$ & $\mathrm{~cm}$ & Particle spherical diameter equivalent \\
\hline$\Delta P$ & $\mathrm{gcm}^{-1} \sec ^{-2}$ & Pressure drop along column \\
\hline$d_{p m}$ & $\mathrm{~cm}$ & Average particle diameter (measured) \\
\hline$k_{0}$ & None & Guiochon's residual reciprocal permeability coefficient \\
\hline$P_{t}$ & None & Guiochon's modified permeability coefficient in Kozeny-Blake equation \\
\hline$L$ & $\mathrm{~cm}$ & Column length \\
\hline$P_{0}$ & None & Permeability coefficient in Kozeny-Blake equation \\
\hline Q & $\mathrm{cm}^{3} \min ^{-1}$ (exception) & Volumetric fluid flow rate \\
\hline$t_{0}$ & $\sec$ & Time for elution of totally permeating unretained solute \\
\hline$k$ & $\mathrm{~cm}^{2}$ & General permeability coefficient in Darcy equation \\
\hline$K_{F}$ & $\mathrm{~cm}^{2}$ & Halász's permeability coefficient in Darcy equation (1974 paper) \\
\hline K & $\mathrm{cm}^{2}$ & Halász's permeability coefficient in Darcy equation (1972 paper) \\
\hline$K_{c}$ & None & Guiochon's permeability coefficient in the Kozeny-Blake equation (as modified by Carmen) \\
\hline \multicolumn{3}{|l|}{ Greek } \\
\hline$\varepsilon_{0}$ & None & External column porosity \\
\hline$\varepsilon_{i}$ & None & Internal column porosity \\
\hline$\varepsilon_{t}$ & None & Total column porosity \\
\hline$\varepsilon_{p}$ & None & Particle porosity \\
\hline$\Phi$ & None & Giddings' ratio of external and total column porosity \\
\hline$\phi$ & None & Flow resistance parameter based on mobile phase velocity \\
\hline$\phi_{0}$ & None & Flow resistance parameter based on superficial velocity \\
\hline$\Omega_{p}$ & None & Particle sphericity factor \\
\hline$\eta$ & $\mathrm{gcm}^{-1} \sec ^{-1}$ & Fluid absolute viscosity \\
\hline$\mu_{i}$ & $\mathrm{cmsec}^{-1}$ & Average fluid interstitial linear velocity \\
\hline$\mu_{s}$ & $\mathrm{cmsec}^{-1}$ & Average fluid superficial linear velocity \\
\hline$\mu_{t}$ & $\mathrm{cmsec}^{-1}$ & Average mobile phase velocity \\
\hline$\Psi$ & None & Porosity dependence term in the Kozeny-Blake equation based on mobile phase velocity \\
\hline$\Psi_{0}$ & None & Porosity dependence term in the Kozeny-Blake equation based on superficial velocity \\
\hline
\end{tabular}

raw data for column $B_{2}$, are supportive of the value of 267 for $P_{0}$.

9.2. Guiochon's 1999 Study. In a 1999 publication by Farkas et al. entitled "Validity of Darcy's Law at Low Flow Rates in Liquid Chromatography" [20], Guiochon and his coauthors report the results of some very exacting measurements which they made to validate Darcy's law at extremely low fluid flow rates. Using a column containing particles packed to a measured external column porosity of 0.399 and having a measured total column porosity of 0.593 (Figure 2 in the paper), Guiochon et al. measured the column pressure drop and fluid flow rate at flow rates ranging between 0.015 and $0.5 \mathrm{~mL} / \mathrm{min}$ with ethylene glycol as the fluid. The authors state that the particles in the column are spherical and they appear to have gone to extraordinary lengths to obtain an accurate measure of the particle diameter and, of course, as a result of modern techniques of particle size classification, the particle size distribution is very narrow.

In Figure 1 of the paper, the authors show a plot of column measured pressure drop in bar versus "fluid linear velocity" in $\mathrm{cm} / \mathrm{sec}$. This plot represents their measurements taken in the empty column. The only velocity that exists in an empty column is the superficial velocity and, thus, the abscissa values in the plot in Figure 1 designated as "fluid linear velocity" represent superficial linear velocity.

In Figure 2 in their paper, however, the authors show another plot of measured pressure drop in bar, also versus "fluid linear velocity" in $\mathrm{cm} / \mathrm{sec}$. The velocity on the abscissa in this plot, although also designated as "fluid linear velocity," does not equate (for permeability related purposes) to the "fluid linear velocity" in Figure 1. This is because in Figure 2, the column was filled with porous particles and the measured velocity was the mobile phase velocity. Indeed, the only velocity used throughout the entire paper is the mobile phase velocity (mobile phase velocity and superficial velocity are one and the same in an empty column).

In Table 1 of their paper, the authors report that for the column represented in Figure 2, the slope of a line achieved when the mobile phase velocityin units of $\mathrm{cm} / \mathrm{sec}$ is plotted versus pressure drop in units of bar is 1829. They also report that the flow resistance parameter, $\phi$, for this column is 865 . In Table 3 herein, it can be seen that the raw data for this column (Column C) generates an apparent value of 257 for $P_{0}$. This value is very close to Giddings' value of 267. Indeed, by referring to Figures 2 and 3 herein, one can see that the reported raw data in this study, without any modification 
whatsoever, essentially confirms our frame of reference in all respects.

Although unnecessary because the small discrepancy in the values of $P_{0}$ could result from experimental error in almost any of the measurements, we believe that even that can be explained. Because of the extraordinarily sensitive nature of the porosity dependence term in the Kozeny-Blake equation, it is suggested herein that this small difference arises from, on the one hand, the authors' technique of using a mixture of methanol and water as the fluid for their determination of the value of $\varepsilon_{t}$ and their use of dichloromethane as the fluid in their determination of the value of $\varepsilon_{0}$ and, on the other hand, their use of ethylene glycol as the fluid when measuring the column pressure gradient. A small difference in the wetting characteristics of these three fluids could easily disrupt the balance between the measured values for $\phi$ and $\Psi$ and, accordingly, account for all the discrepancies. Therefore, in the next row of Table 3 , we make a correction for column pressure to account for this discontinuity in the authors' experimental protocol. Note that the corrected value is an adjustment upward of about $4 \%$ which is within the experimental error of the measurement. Finally, the corrected data for this column establishes a value for $P_{t}$ of 158 which is indicative of an internal porosity for these particles that is known to be slightly higher than the Nova Pak particles reported in Guiochon's 1998 paper. The care with which the authors made their measurements of particle size and flow rates coupled with the measured value of approximately 0.4 for $\varepsilon_{0}$, again a reasonable value for a wellpacked column, makes this study of column permeability one of the most credible and most important in the published literature.

9.3. Guiochon's 2006 Study. In a 2006 publication with Fabrice Gritti, entitled "Experimental Evidence of the Influence of the Surface Chemistry of the Packing Material on the Column Pressure Drop in Reverse-phase Liquid Chromatography" [21], Guiochon and his coauthor claim to make what one can only characterize as a startling revelation. In their application of what they call the "Kozeny-Carman equation," they assert that their data support a value of 97.5 for the Kozeny-Carman "constant," which they acknowledge is "nearly one-half the value traditionally accepted." Moreover, the authors go on to suggest-equally surprisingly-that the nature of the chemical coating on the surface of the particles adds another variable to the relationship between pressure gradient and fluid flow. We show the data for these columns as our series D in Table 3 herein. One can immediately see in Figure 3 herein that the raw data reported for these columns does not resemble that of packed columns in any shape or form. In fact, the data is so bizarre that it falls outside all the boundaries of our frame of reference.

To begin with, the authors used a novel procedure to determine the external porosity of the columns which is based upon the so-called Donnan Effect. Unfortunately, the authors did not include, for comparison purposes, a determination of the external column porosities by some conventional technique. In view of this lack of cross-validation, one is automatically skeptical of the very low values which they derived for the external column porosities.

The authors recite the following in their paper: "the columns used in this work are the same as those the adsorption properties of which were reported earlier (reference included)." The reference was to another paper published in the same year by the same authors [22] in which the total porosity, $\varepsilon_{t}$, of these same columns was measured and reported in Table 1. It is mystifying, therefore, that the authors ignored these measurements in their analysis of permeability reported in this paper. In Table 3 herein, the values from this earlier paper for total column porosity for each of the columns in the study are included. As can be seen in the table, the difference between the total column porosities reported for these columns in the earlier study and the external column porosities reported for them in the present study would indicate internal column porosities which are far too large for these particles when compared to the low values of $P_{t}$ dictated by the measured pressure drops Similarly such internal column porosities would be at significant odds with the specifications published by the manufacturer for these particles. The results of ISEC (inverse size exclusion chromatography) measurements on a standard $3.9 \times 150 \mathrm{~mm}$ Symmetry column were reported by the manufacturer as $\varepsilon_{i}=0.265$, whereas the measurements by the authors would establish a range of values for the internal column porosities from 0.24 to 0.43 .

The other thing to note about this study is that the columns that the authors used in the study were "prototype columns" which had all been "packed and generously offered by the manufacturer (Waters, Milford, MA, USA)" [21, p. 193] and, rather than measuring the particle diameters themselves, the authors merely accepted the nominal particle size given by the manufacturer. This is a significant deviation from Guiochon's standard operating procedure described in his 1999 paper discussed above, in which Guiochon and his coauthors went to extraordinary lengths to measure accurately the particle size, underscoring its importance as follows: "the nominal particle sizes given by manufacturers of silica adsorbents used in chromatography are often approximate averages which cannot be used for accurate calculations of column permeability. For this reason, we measured the particle size distribution by laser-beam scattering, using a Mastersizer instrument (Malvern Instruments, Southborough, MA. USA)" [20, p. 41] (emphasis added). In defense of their failure to do likewise in this 2006 paper, Guiochon and Gritti state the following: "since we did not empty the columns, we had no access to the inner diameter. We measured the external diameter of the tube instead" [21, p. 196]. Although the condition that they not open the columns was presumably imposed by the manufacturer, this does not obviate the need for the authors to have obtained some independent verification of the particle size, especially since it is such a sensitive parameter in the pressure drop/fluid flow relationship. ${ }^{9}$

It can be seen from Table 3 that both the nominal particle size reported by the manufacturer and the external porosities measured by the authors were too low. For example, looking at Figure 3 herein, it is obvious that the raw data values for 
$\Psi$ are greater than the maximum in our reference charts for packed columns. Similarly, Figure 2 herein illustrates that the values for $P_{t}$ are too low and do not reflect values for columns packed with fully porous particles. Both the particle size and the external porosity are suspects. Accordingly, we deduce that the particle size and value for $\Phi$ need to be adjusted. As for the particle size, we adopt the value of 5.5 micrometers, a reasonable deviation from the nominal size given by the manufacturer. Then, using Giddings' value of 267 for $P_{0}$, we show the impact upon internal column porosity due to variations in the level of surface modified stationary phase.

The corrected data all makes sense. The column internal porosity is at its highest for Column $\mathrm{D}_{1}$ which contained underivatized silica particles. This is corroborated by the value of $P_{t}$, which is also at its highest for this column. On the other hand, both these two parameters are at their lowest values for Column $\mathrm{D}_{6}$, which is consistent with the highest coating of $\mathrm{C}_{18}$ stationary phase and is the more typical value for commercially available reverse phase $\mathrm{C}_{18}$ columns. Additionally, the corrected column porosities are consistent with reported values for standard symmetry columns sold by Waters and are consistent with a professionally developed slurry column packing protocol. Finally, the range of corrected $\phi$ values is totally consistent with what one would expect.

Finally, it should be noted that in the very next year after they published this paper, these same authors published another paper on column permeability, which is examined below, in which they discontinue the use of the Donnan Effect to measure column external porosity and where they revert to using the ISEC technique. ${ }^{10}$ In essence, then, even Guiochon himself has effectively abandoned this procedure, but, paradoxically, he still clings to the erroneous conclusions based upon its use (see his comments in his 2010 paper, reviewed below, concerning the allegedly embedded unidentified variables in the value of $K_{c}$ ).

9.4. Guiochon's 2007 Study. In a 2007 publication with coauthors Gritti et al. entitled "Comparison Between the Efficiencies of Columns Packed with Fully and Partially Porous $\mathrm{C}_{18}$-bonded Silica Materials" [23] (Column E series in Table 3 herein), Guiochon discusses the pressure drop/fluid flow relationship in terms of the "Kozeny-Carman equation." In this paper, the authors compare the permeability of two different types of columns, one set filled with fully porous particles and the other set filled with pellicular or partially porous particles, the latter of which they claim are representative of a new paradigm in column/particle design aimed at the more challenging modern-day chromatographic applications where speed of analysis combined with high efficiency require the use of very high total column pressures.

In Figure 4 of their paper, the authors display two values for $K_{c}$, which they define as the "Kozeny-Carman constant." The value of 250 supposedly corresponds to the permeability data set for the columns containing the partially porous particles and the value of 165 supposedly corresponds to the permeability data set for the columns containing the fully porous particles. Although the plot shows a total of 13 data points, the methodology used to derive the values for the constant on the ordinate of the plot is blind to the reader. In other words, the authors do not show any connection between the measured column pressures and the ordinate values in their Figure 4 for the values of the "constant." Moreover, although the caption under their Figure 4 states that the fluid used was acetonitrile/water/TFA $(60 / 40 / 0.1 \%$, $\mathrm{v} / \mathrm{v})$ at $T=295 \mathrm{~K}[23$, p. 297], none of the pressure data displayed in their Figure 2 matches this combination of fluid temperature and eluent compositions. Accordingly, it must be concluded that the measured column pressures upon which the calculated values for the constant in their Figure 4 are based are omitted from the paper.

The authors conclude that "the Kozeny-Carman constant of the Halo column is approximately 250 while that of the other column is close to 170, typical of the result found for conventional beds of spherical porous silica particles $\left(K_{c} \approx\right.$ 180 ). The much larger value of $K_{c}$ for the Halo material is unexpected" [23, p. 295].

Using Figure 2(B) from the paper as a reference, it is apparent that the values of 165 and 250 for $K_{c}$ as displayed in their Figure 4 do not compute. These values for the Kozeny-Carman constant would produce values of 230 and 254 bar, respectively, for the total column pressure at a fluid volumetric flow rate of $3 \mathrm{~mL} / \mathrm{min}$ which corresponds to the authors' value for $u_{s}$ (which they display in Figure 2(B) as being $\left.3 \mathrm{~mm} / \mathrm{sec}^{-1}\right)$. Surprisingly, these values are less than those plotted in Figure 2(B). Therefore, the values of 165 and 250 cannot be representative of the value of $P_{0}$ for this data set of measured column pressures. Moreover, the mobile phase which underlies their values for $K_{c}$ was at a temperature of $22^{\circ} \mathrm{C}(295 \mathrm{~K})$, which is even lower than that used in Figure 2(B), that is, $60^{\circ} \mathrm{C}$. Accordingly, the column pressures which support the $K_{c}$ values in their Figure 4 must be significantly greater than 300 bar at this flow rate (fluid viscosity increases as temperature decreases).

Table 3 herein contains the raw data for both columns reported in the paper and is displayed as columns $E_{1}$ and $\mathrm{E}_{2}$. Referring again to our Figures 2 and 3, it is clear that the authors mistook $P_{t}$ for $P_{0}$. Accordingly, we show the authors' values for $K_{c}$ in Table 3 as being values for $P_{t}$, not $P_{0}$. Thus, corrected, the raw data for the fully porous particles generate a value of 165 for $P_{t}$ and an apparent value of 256 for $P_{0}$. However, the measured value for external porosity of 0.3830 is still on the low end of what is typical for well-packed columns using slurry packing. In addition, this would dictate a value of 0.425 for particle porosity, a value which does not make sense (too high) given the high pressure under which these particles must be slurry packed in order to sustain their very high operating pressures. It was the same high particle porosity, for instance, in Guiochon's 1998 study reviewed above, that caused the irregular particles in that study to crush under the hydraulic pressure of the slurry packing process used therein. Accordingly, we show an adjusted value of 0.4 for external porosity and as dictated by the microscopy results displayed in the paper for these particles, a slightly lower average particle size. On the basis of these adjustments, we derive a value for $P_{0}$ of 267 and a value of 172 for $P_{t}$, both of 
which are just what our frame of reference would cause us to expect.

With respect to the partially porous Halo particles, the authors refer to them as being "rugose." Indeed, the electronmicrographs confirm that these particles have a morphology which, in addition to being quasi-spherical, is also rough and scabrous. In other words, they correspond to what Guiochon describes in his textbook as irregular particles. Accordingly, in order to apply the Kozeny-Blake equation (as modified by Carman) to this data set, one must know the value for the spherical particle diameter equivalent of these particles. As displayed in Table 3, the raw data for the partially porous particles generates a value of 250 for $P_{t}$ and an apparent value of 463 for $P_{0}$. When this data is corrected for particle sphericity according to the KozenyBlake equation (as modified by Carman), the result is a value of 0.88 for $\Omega_{p}$ and a corresponding value of 2.1 micrometers for the spherical particle diameter equivalent. The resulting corrected value of 144 for $P_{t}$ is consistent with the low particle porosity which characterizes the Halo particles used in this study.

9.5. Guiochon's 2008 Study. In another paper with Gritti, published in 2008 and entitled "Ultra High Pressure Liquid Chromatography Column Permeability and Changes of the Eluent Properties" [3] (Column F series in Table 3 herein), Guiochon reports a study carried out on four columns which, again, were "generously offered by the manufacturer" [3, p. 2]. In this paper, he and his coauthor draw the conclusion that "the average Kozeny-Carman permeability constant for the columns was $144 \pm 3.5 \%$ " [3, p. 1].

In Table 1 of their paper, Guiochon and Gritti provide a list of column variables, some of which were "given by the manufacturer" and some of which were "measured" in the authors' lab. The authors describe the particles in the columns under study as "fine particles, average $d_{p} \approx 1.7 \mu \mathrm{m}$, of bridged

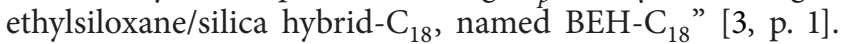
Among the variables which they identify as having been given by the manufacturer is the $1.7 \mu \mathrm{m}$ value for the particle size.

Figure 5 of their paper is a plot of measured pressure drop in bar versus fluid flow rate in $\mathrm{mL} / \mathrm{min}$. In our Table 3 herein, the raw data from the authors' Figure 5 for each of the columns in the study is displayed in the rows for Columns $\mathrm{F}_{1}$ through $\mathrm{F}_{4}$. The computed values for $P_{0}$ are based upon the value of the particle size given in Table 1 of the paper and are the same as those reported by the authors for their columns D1 through D4, namely, 149, 166, 161, and 168. The corresponding values for $P_{t}$ in the raw data average are approximately 100, a value representative of nonporous particles. However, we know that the particles under study are porous because the reported values for $\varepsilon_{t}$ are greater than those reported for $\varepsilon_{0}$. Moreover, the corresponding particle porosity for these columns would be greater than 0.4 which is entirely unreasonable (again too large) for fully porous particles which have to be slurry packed and operated at extremely high pressures (greater than 15,000 psi). Accordingly, as is plainly evident from Figures 2 and 3 herein, there is something fundamentally wrong with this data set.
Since the reported values for total column porosity are not in question, this means that the values for external porosity are again too low and since the average particle size was not measured by the authors, we adjust for these two parameters. Accordingly, in Table 3 herein, a correction is made to thenominal particle size given by the manufacturer and the external porosities are set to the reasonable value of 0.4 . Using the resultant corrected value of about 1.9 micrometers for particle size (slightly higher than the nominal value given by the manufacturer), reasonable values for $\phi$ as well as $P_{t}$ are achieved for all columns in the study.

9.6. Guiochon's 2010 Studies. Next, we consider three more Guiochon studies of permeability which were all reported in 2010 and all of which he again coauthored with Gritti et al. The first of these articles is entitled "Performance of Columns Packed With the New Shell Particles, Kinetex- $\mathrm{C}_{18}$ " [24]. In this study, the authors report permeability results for partially porous particles produced by two different manufacturers. The raw data reported for the two columns are included in our Table 3 as Columns $\mathrm{H}_{1}$ and $\mathrm{H}_{2}$. As reflected in our Table 3 for the raw data, and as is plainly evident from Figure 2 and 2 herein, the resulting values for $P_{t}$ of 96 and 98 are way too low, being more representative of nonporous particles, something which the particles under study are not.

The authors, after having gone into great detail describing what measurements and precautions they used to derive all their experimental measurements, again fail to measure the average particle size. Instead they back-calculate the value for particle size based upon the Kozeny-Blake equation with an embedded value of 180 for $P_{0}{ }^{11}$ Additionally, the external porosity values are once again on the low side. Accordingly, in our Table 3, for comparison purposes, we show corrected values for the particles which are slightly higher than the calculated values. We point out that using a value of 267 for $P_{0}$ and a set value of 0.4 for external porosity establishes values for $P_{t}$ of 142 and 145, values which are very reasonable based upon the authors' own statement of the low particle porosity of these partially porous particles.

The second of Guiochon's 2010 papers entitled “Mass Transfer Resistance in Narrow-bore Columns Packed with $1.7 \mu \mathrm{m}$ Particles in Very High Pressure Liquid Chromatography" [25] further exemplifies the authors' reliance upon manufacturers' data as opposed to measuring things. In this paper, the authors report two columns having the narrow diameter of $2.1 \mathrm{~mm}$. One column contains fully porous particles while the other contains partially porous particles. The authors' data sets are presented in our Table 3 as Columns $\mathrm{H}_{3}$ and $\mathrm{H}_{4}$, respectively. As can be seen from the raw data, and Figures 2 and 3 herein, the results for Column $\mathrm{H}_{4}$ (233 for $P_{0}$ and 121 for $P_{t}$ ) are already essentially in conformance with our frame of reference. Moreover, when we apply a modest adjustment for particle size over that obtained by the author's back-calculation technique, we get even closer to the norm: 265 for $P_{0}$ and 137 for $P_{t}$.

The authors rightfully point out that their ISEC measurements result in higher external porosity values for the column containing partially porous particles, reporting the 
typical value of 0.4090 for the column. However, surprisingly, they stop short of the obvious conclusion that since the technique of ISEC suffers from the limitation of not being able to precisely differentiate between the free space between the particles and the free space within the particles, it is logical that nonporous particles would result in even higher external porosities for these slurry packed columns, representing as they do (nonporous particles, that is) the limit of the phenomenon. Indeed the authors appear to be willing to go to great lengths to justify their selective conclusions avoiding the obvious and more technically relevant explanation which is that the technique which they are using to determine a value for external porosity is flawed and results in too low an external porosity for columns packed with fully porous particles.

Not surprisingly, then, when we turn to the authors' data on the fully porous column in this study, we note that the external porosity is again low. On the other hand, when we set the value for external porosity at the more reasonable level of 0.4 and, again, make a modest adjustment for particle size, we derive the value of 267 for $P_{0}$ and the right-in-the-ballpark value of 167 for $P_{t}$.

The third of Guiochon's 2010 papers, entitled "Performance of New Prototype Packed Columns for Very High Pressure Liquid Chromatography" [26], once again, involves a failure by the authors to accurately assess the contribution of the size of the particles under study to overall pressure drop. The authors use a value of 1.7 micrometers for the particles, the value supplied by the manufacturer of these porous particles.

We have included the raw data reported in this paper in our Table 3 as Columns $I_{1}-I_{6}$. As was the case in Guiochon's other recent studies involving fully porous particles, the column external porosities appear to be understated. Among other things, the reported combination of a high value for column total porosity and a low value for column external porosity dictates high particle porosity. However, these columns and particles are designed to be run at extremely high pressures. Accordingly, the particle porosities need to be low in order for the particles to withstand such high pressures. On the other hand, Guiochon and company report values for $P_{0}$ (which they again notate as $K_{c}$ ) ranging from 139 to 153 . This, in turn, generates values for $P_{t}$ from 91 to 98 , values which, again, when viewed in Figures 2 and 3 herein, are clearly too low because they are representative of nonporous particles. If nothing else, then, the various reported porosities are self-contradictory.

In our corrected values in Table 3, we have corrected for both column external porosity and particle size. It can be seen that an average value of 175 is generated for $P_{t}$, which fairly reflects the porosity of these particles (as described by the authors themselves in their Table 1).

9.7. Guiochon's 2011 Study. Finally, we come to Guiochon's 2011 publication relevant to our topic, yet another paper he coauthored with Gritti. This paper is entitled "The Mass Transfer Kinetics in Columns Packed with Halo-ES Shell Particles" [27]. This study involved two columns of different particle porosities. One column contained particles having a particle porosity, $\varepsilon_{p}$, of 0.16 while the other contained particles of porosity 0.27 . The authors measured the average particle size for each column using SEM which resulted in particle sizes of 2.87 and 3.02 micrometers for the respective columns. The pressure drop for each column was measured in two different mixtures of Acetonitrile Water, thereby generating two data points for each column. Both columns in this study generated typical values of 0.4 for external porosity. The results are presented in Figure 3 in their paper. In Table 3 herein, the results of the raw measurements are presented as our Columns $\mathrm{J}_{1}$ and $\mathrm{J}_{2}$. The values of $P_{0}$ (which, yet again, Guiochon and Gritti notate in their paper as $K_{c}$ ) corresponding to the lower particle porosity column $\left(\mathrm{J}_{1}\right)$ are 234 and 232 while values for the higher particle porosity column $\left(\mathrm{J}_{2}\right)$ are 289 and 273 . The average of these four measured values is 257 .

Recognizing, finally, that their data indicates that Carman's value of 180 for $P_{0}$ may be too low, the authors proceed to challenge their own data. Singling out the highest value of $P_{0}, 289$, the authors comment that such a high value could be explained by a clogging of the column end frit. However, they also report that they adjusted for extra-column effects in their reported pressure drop values. Since this procedure presumably required measurements in the empty columns, one would think that this would have provided the necessary adjustments to eliminate the possibility of a clogging issue. Moreover, even if the highest value for $P_{0}$ represents a clogged frit, it is unlikely that the other value for the same column taken in the other fluid-which, at 273, was almost as highalso represented a clogged frit. And then, of course, there are the two data points for the other column, 234 and 232.

Lacking any other explanation for these unexpectedly (from their point of view) high values of $P_{0}$, they jettison their own measured values for particle size and, instead, opt for the manufacturers' lower values of 2.7 micrometers. This lower particle size, of course, results in the lower calculated values for $P_{0}$. As reflected in Figure 3 of their paper, they are now able to report values for $P_{0}$ of 231 and 218 for the higher particle porosity column and 207 and 206 for the lower particle porosity column.

As can be seen from Table 3 herein, when permeability measurements are used to determine particle size, a single value for $P_{0}$ of 267 for all four data points defines a particle size range of 2.90-3.08 micrometers, which is almost exactly what the authors actually measured by SEM. Before he rejected his own SEM measurements, Guiochon obtained an average value of 257 for $P_{0}$. Suffice it to say that his value is significantly closer to the expected (by us) value of 267 than it is to 180 . Moreover, if one makes a reasonable allowance for experimental error, one could say that Guiochon's 2011 study essentially confirms that the value of $P_{0}$ is 267 and, accordingly, he confirms our permeability frame of reference. ${ }^{12}$

\section{Conclusions}

Giddings' teaching of column permeability in columns packed with fully porous particles is based upon a calibration 
derived from nonporous particles which has been titrated for porous particles based upon independently derived data for particle porosity via his $\Phi$ parameter, whereas Guiochon's teaching is not. This is the fundamental reason for the discrepancy between their respective teachings on column permeability. Guiochon's textbook teaching is based upon a term derived from the chromatographic literature-the flow resistance parameter, $\phi-$ which has its roots in thermodynamic theory rather than hydrodynamic theory. In addition, the fact that Guiochon based his worked example on particles having an irregular morphology $\left(k_{0}=1.0 \times 10^{-3}\right)$ without specifying the degree of irregularity of the particles embedded in his hypothetical value for column pressure, rather than basing it upon smooth, spherical particles where the characteristic dimension of the particle is well-defined and contains no ambiguity with regard to particle morphology, almost makes it appear as if he was deliberately leaving himself some wiggle room in his teaching.

But there can be no wiggle room in the value of $P_{0}$. To begin with, as modified by Carman to accommodate particles with an irregular shape, the Kozeny-Blake equation specifically accounts for particle morphology within the realm of streamline flow. Moreover, since the relationship in the equation between pressure gradient and fluid velocity depends, to a first approximation, on the fourth power of the column external porosity, the value of $P_{0}$ must be both accurate and precise. This degree of accuracy and precision, however, can only be realized experimentally when all variables are accurately measured; in which case, the values for column pressure embedded in the flow resistance parameter, $\phi$, on the one hand, and the value for column total porosity embedded in the porosity dependence term, $\Psi$, on the other hand, are self-calibrating. ${ }^{13}$

Moreover, Guiochon's occasional (albeit apparently unwitting) publication of the value of the modified permeability coefficient $P_{t}$, as if it were the value of $P_{0}$, has only exacerbated the confusion surrounding the value of $P_{0}$. As has been demonstrated herein, experimentally derived values of 180 and 150 for $P_{t}$ are not infrequent for columns packed with porous particles and, accordingly, may unfortunately be confused with the values of Carman $\left(P_{0}=180\right)$ and Ergun $\left(P_{0}=150\right)[28] .{ }^{14}$

As detailed above, a recurrent theme in Guiochon's experimental studies of packed bed permeability over the last decade or so has been that Carman was right: $P_{0}$ is a constant and its value is 180 . Accordingly, when the results of his experiments have not supported this theme, Guiochon and his coauthors have seemingly gone out of their way to attempt to produce results that are consistent with his predetermined worldview. The devices by which this has been pursued include (a) ignoring measured particle size data in favor of the particle manufacturer's stated size, (b) not measuring particle size at all and back-calculating particle size from permeability measurements where a value for $P_{0}$ of 180 has been plugged into the Kozeny-Blake equation as a given, and (c) hypothesizing unrealistic explanations for the discrepancy such as the existence of a "clogged end frit."

On the other hand, facts being the stubborn things that they are, Guiochon's efforts to make the results of his experiments fall into line have not infrequently failed to meet with success.

However, instead of considering the obvious possibility that Kozeny-Blake is valid and that $P_{0}$ is indeed a constant, but that its value is not 180 , Guiochon has hedged his bets by reverting back to the ambiguity (and safety) of Darcyism. For example, even after Guiochon does a complete flip-flop in his review paper from his textbook teaching and-without any explanation or analysis-signs on to the conventional wisdom that the value of $P_{0}$ is 180, as if taking one step forward and two steps backward, he proceeds to announce: "there is some uncertainty on the value of the numerical coefficient but since few authors report column permeability data, a systematic study has not been made yet" [14, p. 9].

Likewise, in the second of their 2010 papers which we reviewed above, Guiochon and his frequent coauthor Gritti make this illuminating statement "the external porosity, $\varepsilon_{e}$, of packed beds is an important characteristic of HPLC columns because it affects the column permeability, which is essentially controlled by the average particle size, $d_{p}$. The shape of the packed particles, their size distribution, and the chemical nature of their external surface play also a significant role in the column permeability but their impact is more difficult to assess. Their effect is empirically lumped into the Kozeny-Carman constant, $K_{c}$ " [21, p. 1487] (emphasis supplied). Suffice it to say that this assertion that $P_{0}$ is nothing but a hodge-podge of unidentified variables is totally inconsistent with the notion that it is a constant with a fixed value of 180 .

Ironically, Guiochon has essentially ignored his own study published in the 1999 paper with Farkas and Zhong which represents one of the most credible studies of column permeability available in the literature and which contradicts both extremes of his pronouncements on column permeability: (a) there is a constant and its value is 180 and (b) there is only a residual permeability coefficient which is a variable number that one must plug in to balance the two sides of the pressure/flow equation. Indeed, it is our conclusion that Guiochon has actually ignored all of his experimental investigations, which, when appropriately understood, uniformly corroborate Giddings' conclusion that the value of $P_{0}$ is 267; a conclusion which was implicit in the first edition of his textbook published in 1965 and became explicit in the second edition published in 1991 [29, p. 65].

\section{Conflict of Interests}

The authors declare that there is no conflict of interests regarding the publication of this paper.

\section{Acknowledgment}

Sincere gratitude is expressed to Tivadar Farkas for providing the raw measurements underlying the study reported in Guiochon's 1999 paper, of which Farkas was a coauthor. 


\section{Endnotes}

1. Endnotes The terminology "Kozeny-Carman equation" is not favored by the current authors. This is because it represents the Kozeny-Blake equation with a value for $P_{0}$ of 180 which is attributed to Carman and which is believed by us to be in error. Accordingly, the terminology "Kozeny-Blake equation (as modified by Carman)" is preferred and represents Carman's contribution to the equation to accommodate nonspherical particles, which is a legitimate modification.

2. For an in-depth review of Giddings' development of this parameter, see [1] herein.

3. If the column length is in $\mathrm{cm}$, the particle diameter in $\mu \mathrm{m}$, the viscosity in $\mathrm{cP}$, and the pressure drop in psi, our equation becomes

$$
\Delta P(\mathrm{psi})=\frac{15 u(\mathrm{~cm} / \mathrm{s}) \eta(\mathrm{cP}) L(\mathrm{~cm})}{k_{0} d p^{2}\left(\mu \mathrm{m}^{2}\right)} .
$$

As an example of calculation, assume the following values: linear velocity $0.10 \mathrm{~cm} / \mathrm{s}$, viscosity, $1.0 \mathrm{cP}$, column length $15 \mathrm{~cm}$, particle size $10 \mu \mathrm{m}$, and permeability constant $1 \times 10^{-3}$. The pressure drop is

$\Delta P=\frac{15[0.10 \mathrm{~cm} / \mathrm{s}][1.0 \mathrm{cP}][15 \mathrm{~cm}]}{\left[1.0 \times 10^{-3}\right]\left[10^{2}\right]}=225 \mathrm{psi}$.

Note that we have corrected two obvious typographical errors in the worked example: (1) the value of one parameter used in $(5.87 \mathrm{~d})$ compared to the statement of that value in Guiochon's text (15 instead of $25 \mathrm{~cm}$ for the length of the column) and (2) the value of the calculated pressure drop (225 instead of $325 \mathrm{psi}$ ).

4. The data in Tables 1 and 2, both of which are reference charts, are based upon a column of the length $(15 \mathrm{~cm})$, packed with particles of the size (10 micrometers), with fluid of the viscosity $(1.0 \mathrm{cP})$ and running at the mobile phase velocity $(.10 \mathrm{~cm} / \mathrm{sec})$ specified in Guiochon's worked example.

5. This was the technique used by Giddings to establish the value of 267 for $P_{0}$ which is implicit in Table 5.3-1 in his 1965 text [1] and [8, p. 209].

6. It also leads to an apparent value for Guiochon's coefficient $P_{t}$ of 178 . Note the coincidental and unfortunately confusing agreement between this value and Carman's much touted value of 180 for $P_{0}$ [6].

7. It also generates a value of 148 for $P_{t}$. Again, note the confusing coincidence between this value and the value of 150 which is also frequently reported in the literature as being the value of $P_{0}[10,11]$.

8. Neue uses the terminology of 1,000 and $1 \times 10^{-3}$ interchangeably, apparently, in his handbook. This practice is sometimes used throughout the literature as the "constant" in the Kozeny-Blake equation may be thought of as either a reciprocal coefficient or a coefficient of direct proportionality depending on the author's accepted convention. For instance, this is why we refer to Guiochon's use of his term $k_{0}$ as a "reciprocal" coefficient.

9. Additionally, since the authors did not have firsthand knowledge of the value of either the particle size or the tube inner diameter, their conclusion that "the residual explanation is that the interstitial velocity distribution between the packed particles depends on the chemical nature of the external surface of these particles" is unjustified.

10. On the other hand, it is widely accepted that, at least when practiced with the currently available less than adequate solute standards, the ISEC technique does not provide an accurate differentiation between pores within and without the particle fraction of a chromatographic column containing fully porous particles. Beginning around 2007, Guiochon compounded this problem by changing his method of interpreting ISEC curves. The conventional method had been to use the intersection of both branches of the ISEC curve [12] and [10, p. 143]. Guiochon is now extrapolating a single branch to zero elution volume. In addition, he is now using "holdup" volumes measured on a gravimetric basis to establish values for total column porosity. Both of these practices may be contributing to even lower external porosity values for fully porous particles from those that would be obtained by using traditional ISEC protocols.

11. Even more surprising, the authors reference Giddings' 1965 text as authority for their chosen value of 180 for $P_{0}$. This is a clearly erroneous citation of Giddings' 1965 teaching on permeability. While Giddings stated in his 1965 text that the most commonly referenced value for $P_{0}$ in the Kozeny-Blake equation was indeed 180, he rejected this value in favor of a much higher value for $P_{0}$. He did this by using his $\phi^{\prime}$ parameter in his own permeability equation, thereby establishing that his measured values were in complete disagreement with Carman's value. Moreover, he also stated that Carman's discrepancy had also been identified by Giddings [8, p. 208].

12. Our discussion of Guiochon's publications ends with this 2011 paper. The present authors have decided not to critique each and every Guiochon paper ever since 2011 - of which there have been several — in which Guiochon and his collaborators state a value for $P_{0}$ (or as he calls it, $K_{c}$ ). However, that is due to the following decisions by the present authors: (a) enough is enough, (b) many of the assertions in those papers of values for what should be measured parameters are apparently assumed, not measured and are, in general, opaque to and indeterminable by the reader, and (c) these papers, like their recent predecessors, claim in the introductory narrative that the value of $P_{0}$ is 180 , but then go on to record supposedly different experimental values for $P_{0}$, without ever reconciling the two.

13. On the other hand, even carefully constructed and controlled experiments can take us only so far. We have used 267 in this paper as the value of the constant in the Kozeny-Blake equation. That is the number that we 
calculated from the results which Giddings obtained from his experiments conducted in 1965, over forty years ago [1]. Giddings himself, in the 1991 edition of his textbook, rounded his results off at the figure of 270 [29, p. 65]. We have no doubt that the exact value of the constant is somewhere between 265 and 270 and, therefore, we feel very comfortable in using 267, which is the average of those two values, in our analysis of Guiochon's teaching and his recent experimental work. However, a definitive determination of the constant's value will have to await its theoretical development from first principles, something which has never been done.

14. It is also possible that this type of mistaken identity may have infected the work of some of the other contributors to the hydrodynamic literature.

\section{References}

[1] H. M. Quinn, "Reconciliation of packed column permeability data-part 1: the teaching of Giddings revisited," Special Topics \& Reviews in Porous Media, vol. 1, no. 1, pp. 79-86, 2010.

[2] H. M. Quinn and J. J. Takarewski, "High performance liquid chromatography method and apparatus," US Patent no. 5,772,874, 1998.

[3] F. Gritti and G. Guiochon, "Ultra high pressure liquid chromatography. Column permeability and changes of the eluent properties," Journal of Chromatography A, vol. 1187, no. 1-2, pp. 165-179, 2008.

[4] F. Gritti and G. Guiochon, "Adsorption mechanism in reversedphase liquid chromatography. Effect of the surface coverage of a monomeric C18-silica stationary phase," Journal of Chromatography A, vol. 1115, no. 1-2, pp. 142-163, 2006.

[5] G. Guiochon, S. G. Shirazi, and A. M. Katti, Fundamentals of Preparative and Nonlinear Chromatography, Academic Press, Boston, Mass, USA, 1994.

[6] P. C. Carman, "Fluid flow through granular beds," Transactions of the Institution of Chemical Engineers, vol. 15, pp. 155-166, 1937.

[7] L. R. Snyder and J. J. Kirkland, Introduction to Modern Liquid ChromaTography, John Wiley \& Sons, 2nd edition, 1979.

[8] J. C. Giddings, Dynamics of Chromatography, Part I: Principles and Theory, Marcel Dekker, New York, NY, USA, 1965.

[9] G. Guiochon, S. G. Shirazi, and A. M. Katti, Fundamentals of Preparative and Nonlinear Chromatography, Academic Press, Boston, Mass, USA, 2nd edition, 2006.

[10] S. Ergun, "Fluid flow through packed columns," Chemical Engineering Progress, vol. 48, pp. 89-94, 1952.

[11] R. B. Bird, W. E. Stewart, and E. N. Lightfoot, Transport Phenomena, John Wiley \& Sons, 2002.

[12] H. Guan and G. Guiochon, "Study of physico-chemical properties of some packing materials. I. Measurements of the external porosity of packed columns by inverse size-exclusion chromatography," Journal of Chromatography A, vol. 731, no. 12, pp. 27-40, 1996.

[13] G. Guiochon, "Flow of gases in porous media. Problems raised by the operation of gas chromatography columns," Chromatographic Reviews, vol. 8, pp. 1-47, 1966.

[14] G. Guiochon, "The limits of the separation power of unidimensional column liquid chromatography," Journal of Chromatography $A$, vol. 1126, no. 1-2, pp. 6-49, 2006.
[15] U. Neue, HPLC Columns-Theory, Technology and Practice, Wiley-VCH, 1997.

[16] I. Halász, R. Endele, and J. Asshauer, "Ultimate limits in highpressure liquid chromatography," Journal of Chromatography A, vol. 112, no. C, pp. 37-60, 1975.

[17] R. Endele, I. Halz, and K. Unger, "Influence of the particle size $(5-35 \mu \mathrm{m})$ of spherical silica on column efficiencies in highpressure liquid chromatography," Journal of Chromatography A, vol. 99, pp. 377-393, 1974.

[18] I. Halász and M. Naefe, "Influence of column parameters on peak broadening in high pressure liquid chromatography," Analytical Chemistry, vol. 44, no. 1, pp. 76-84, 1972.

[19] J.-H. Koh and G. Guiochon, "Effect of the column length on the characteristics of the packed bed and the column efficiency in a dynamic axial compression column," Journal of Chromatography A, vol. 796, no. 1, pp. 41-57, 1998.

[20] T. Farkas, G. Zhong, and G. Guiochon, "Validity of Darcy's law at low flow-rates in liquid chromatography," Journal of Chromatography A, vol. 849, no. 1, pp. 35-43, 1999.

[21] F. Gritti and G. Guiochon, "Experimental evidence of the influence of the surface chemistry of the packing material on the column pressure drop in reverse-phase liquid chromatography," Journal of Chromatography A, vol. 1136, no. 2, pp. 192-201, 2006.

[22] F. Gritti and G. Guiochon, "Adsorptionmechanism in reversedphase liquid chromatography. Effect of the surface coverage of a monomeric C18-silica stationary phase," Journal of Chromatography A, vol. 1115, no. 1-2, pp. 142-163, 2006.

[23] F. Gritti, A. Cavazzini, N. Marchetti, and G. Guiochon, "Comparison between the efficiencies of columns packed with fully and partially porous C18-bonded silica materials," Journal of Chromatography A, vol. 1157, no. 1-2, pp. 289-303, 2007.

[24] F. Gritti, I. Leonardis, D. Shock, P. Stevenson, A. Shalliker, and G. Guiochon, "Performance of columns packed with the new shell particles, Kinetex-C18," Journal of Chromatography A, vol. 1217, no. 10, pp. 1589-1603, 2010.

[25] F. Gritti and G. Guiochon, "Mass transfer resistance in narrowbore columns packed with $1.7 \mu \mathrm{m}$ particles in very high pressure liquid chromatography," Journal of Chromatography A, vol. 1217, no. 31, pp. 5069-5083, 2010.

[26] F. Gritti and G. Guiochon, "Performance of new prototype packed columns for very high pressure liquid chromatography," Journal of Chromatography A, vol. 1217, no. 9, pp. 1485-1495, 2010.

[27] F. Gritti and G. Guiochon, "The mass transfer kinetics in columns packed with Halo-ES shell particles," Journal of Chromatography A, vol. 1218, no. 7, pp. 907-921, 2011.

[28] M. Rhodes, Introduction to Particle Technology, John Wiley \& Sons, 1998.

[29] J. C. Giddings, Unified Separation Science, John Wiley \& Sons, 1991. 

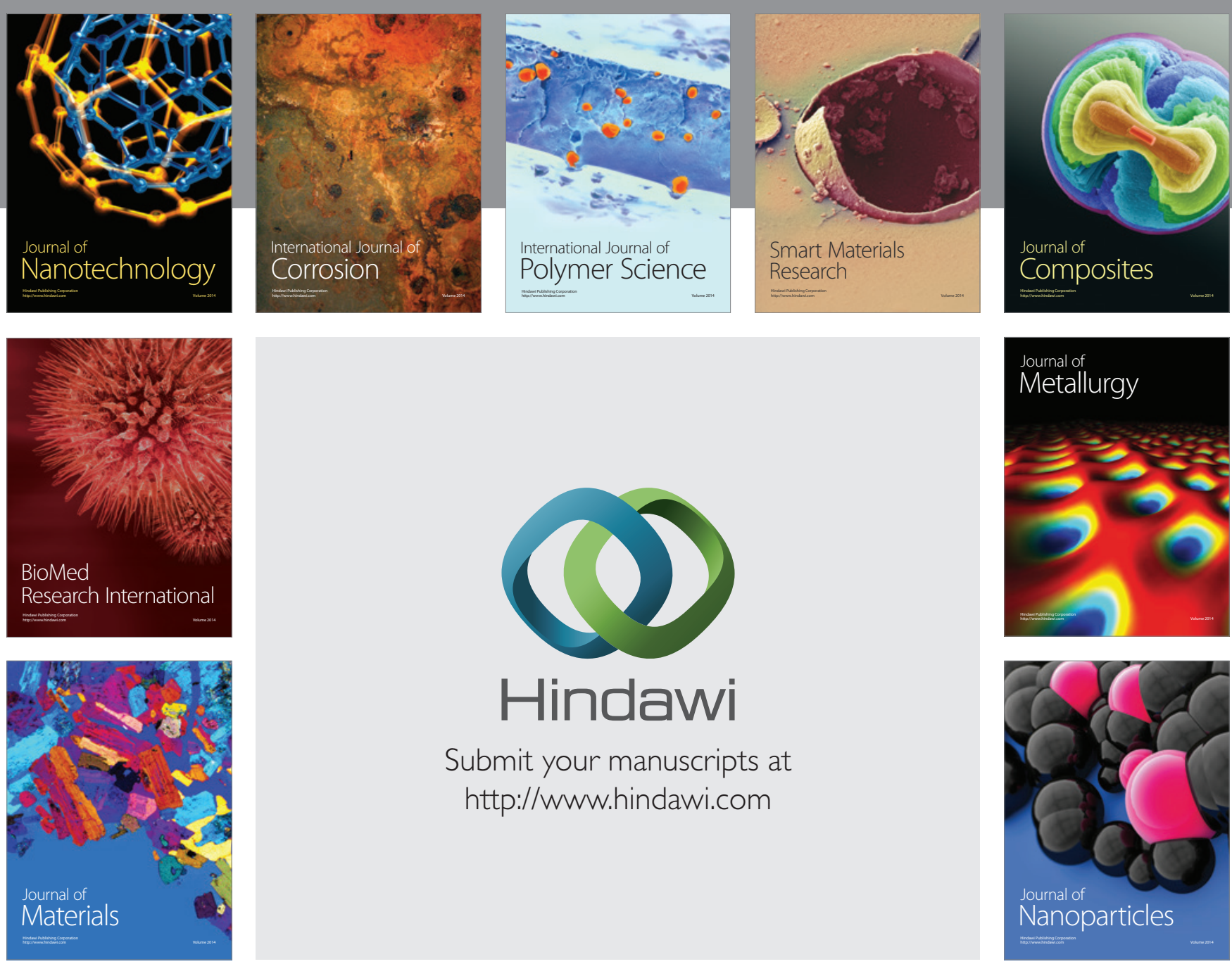

Submit your manuscripts at http://www.hindawi.com
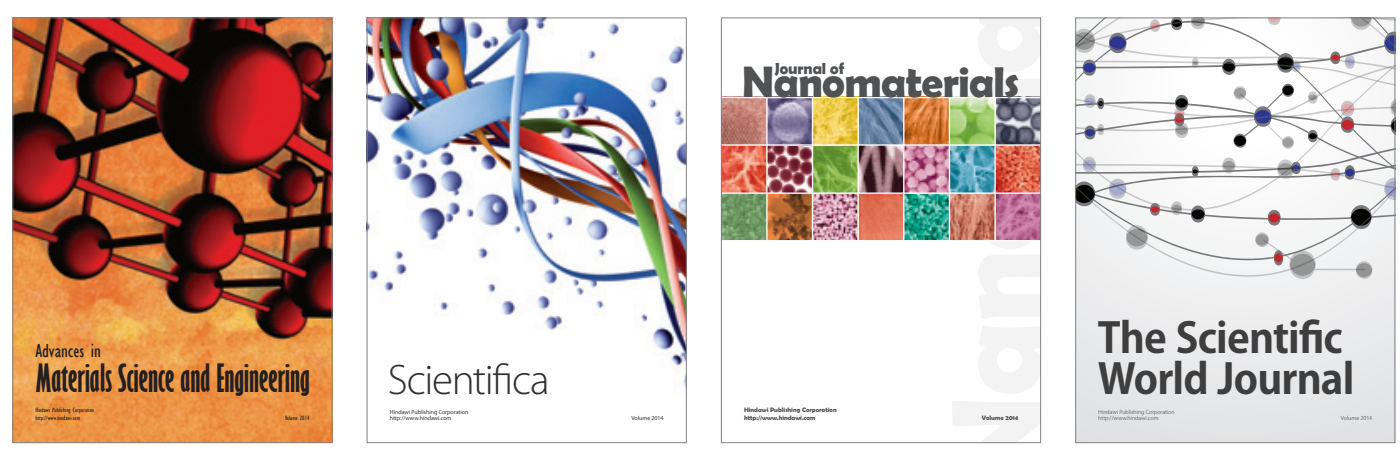

\section{The Scientific World Journal}
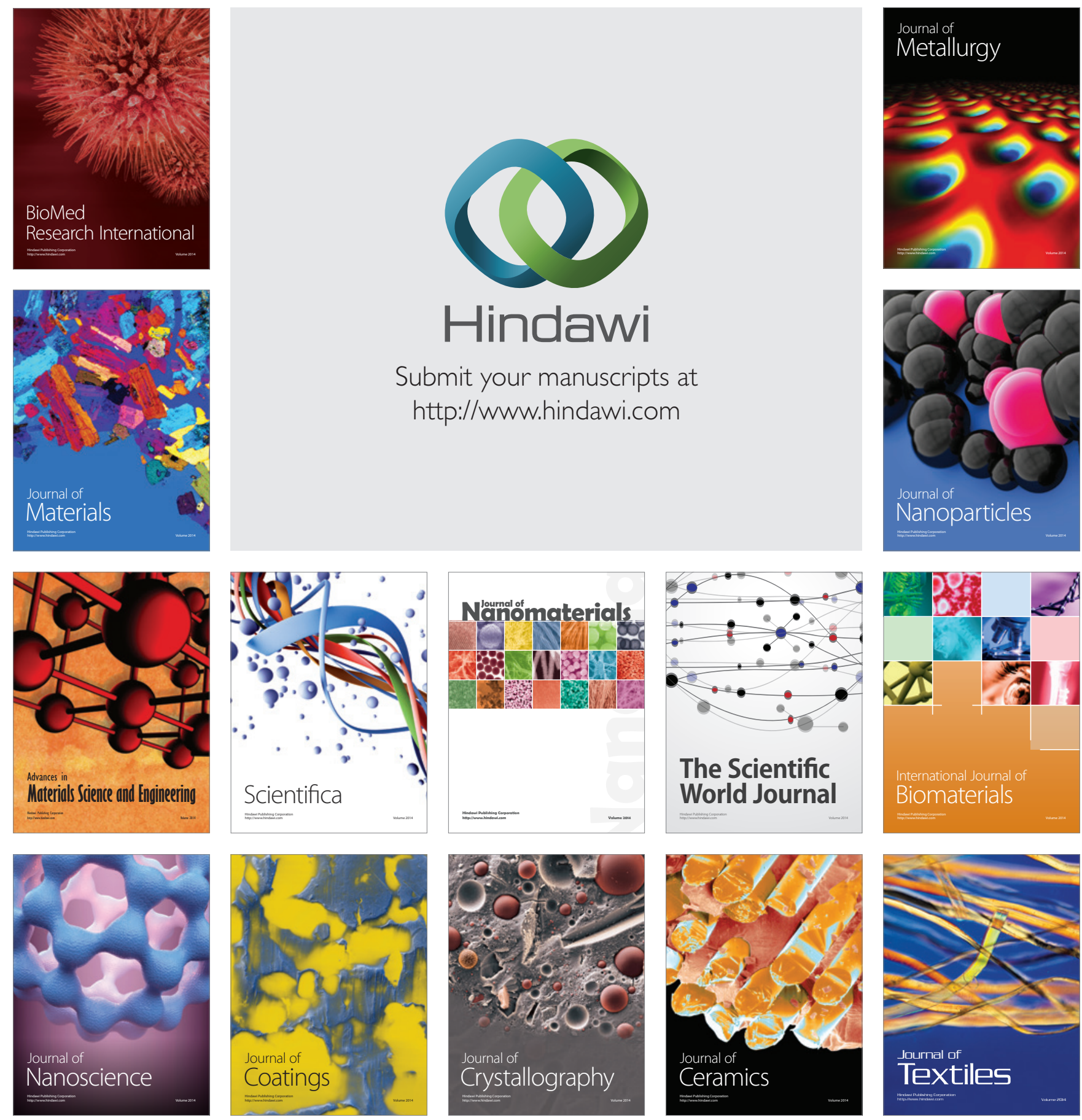